\title{
Variety of Evidence
}

\begin{abstract}
Varied evidence confirms more strongly than less varied evidence, ceteris paribus. This epistemological Variety of Evidence Thesis enjoys widespread intuitive support. We put forward Bayesian models of scientific inference in which we explicate one notion of varied evidence and the Variety of Evidence Thesis by appealing to measures of entropy. Our explication of the Variety of Evidence Thesis holds in all our models which also pronounce on disconfirmatory and discordant evidence. We argue that our models pronounce rightly. Against a backdrop of failures of the intuitive Variety of Evidence Thesis, the case for the Variety of Evidence Thesis emerges strengthened.
\end{abstract}

\section{Introduction}

Varied evidence for a hypothesis confirms it more strongly than less varied evidence, ceteris paribus. In this explicit form, it can be traced back in the philosophy of science at least to (Carnap, 1962, p. 230). It also appears in (Kenynes, 1921, p. 253) "The variety ${ }^{1}$ of the circumstances, in which the Newtonian generalisation is fulfilled, rather than the number of them, is what seems to impress our reasonable faculties", (Horwich, 1982, p. 118) "It is an undeniable element of scientific methodology that theories are better confirmed by a broad variety of different sorts of evidence than by a narrow and repetitive set of data", in (Earman, 1992, p. 77) "It is a truism of scientific methodology that variety of evidence can be as important or even more important that the sheer amount of evidence" and in (Claveau, 2013, p. 94) "Seeking a variety of evidence for a hypothesis is standard practice in science, as well as in normal life".

\footnotetext{
${ }^{1}$ Emphasis original.
} 
Variety of evidence is not only discussed by philosophers but also, for example, in social psychology in (Hüffmeier et al., 2016, p. 82) "The more often a new finding is replicated, and the more different types of replications there are in particular, the more trust and confidence there should be in the validity of this finding" and in epidemiology, by (Borm et al., 2009, p. 711) "The results of a single trial should be interpreted with caution. When it is difficult to predict or determine how trial-specific factors influence the results, the best way to evaluate the performance of a treatment is to use multiple, possibly smaller, trials."

(Hempel, 1966, p. 224) points out that: "For that the confirmation of a hypothesis depends not only on the quantity of the favorable evidence available, but also on its variety: the greater the variety, the stronger the resulting support." Hempel's thinking was given a logical formulation in (Meehl, 1990, p. 109).

Given the predominance of Bayesian reasoning and modelling and the widespread intuitive support for the Variety of Evidence Thesis (VET), one would think that a general Bayesian analysis of the VET comprising of an explication of the notion of varied evidence and the VET has been developed. This, however, is not the case.

\section{The Story of the Variety of Evidence Thesis}

\subsection{The Story - So Far}

While the intuitive appeal of the VET is widely spread, its status within the Bayesian paradigm has been contested. There are three main approaches, the latter two overlap. ${ }^{2}$

The eliminative approach put forward in Horwich (1982) aims to capture the intuition that varied evidence for a hypothesis rules out competing hypotheses better than non-varied evidence, see also (Earman, 1992, p. 79), (Glymour, 1980, pp. 139-142), (Horwich, 1998, pp. 611-613), (Howson and Urbach, 2006, pp. 124-126) and (Schupbach, 2015, p. 314). Schupbach (2017) recently argued that varied evidence does a better job ruling out competitive explanations.

Wayne (1995) extends Horwich's approach from deterministic hypotheses to non-deterministic, i.e., statistical hypotheses and unearths cases in which his version of the VET fails. (Steel, 1996, p. 671) defends the eliminative approach by

\footnotetext{
${ }^{2}$ The notion of evidential variety has received a range of labels, the reader is referred to (Claveau, 2011, p. 249, Footnote 18). Refer to Lloyd (2015); Vezér (2017) for recent Variety of Evidence reasoning in (the rational reconstruction of) climate science.
} 
pointing out that Wayne has not taken a pertinent ceteris paribus condition into account. Fitelson (1996) criticises Wayne (1995) for an uncharitable reconstruction of Horwich (1982). However, Fitelson (1996) finds cases in which the VET fails even in a more charitable reconstruction of the eliminative approach.

In the correlation approach of (Earman, 1992, Section 3.5), see also Franklin and Howson (1984), one considers a body of evidence $\mathcal{E}=\left\{E_{1}, \ldots, E_{n}\right\}$ which is entailed by the agent's background knowledge $K$ and the hypothesis of interest $H$. The variety of the body of evidence $\mathcal{E}$ is greater, the slower $P\left(E_{n} \mid E_{1} \ldots E_{n-1} K\right)$ approaches one. It is then shown that the posterior probability of the hypothesis of interest is greater the greater the variety of the body of evidence. Wayne (1995) later pointed out - correctly we think - that this approach fails to incorporate the prior probabilities of the items of evidence themselves. Adding a ceteris paribus clause requiring that the prior probabilities of the items of evidence to be equal resolves this issue, as argued in Wayne (1995); Myrvold (1996).

Wayne (1995) also alleges that this approach is incomplete as it omits a discussion of how judgements of similarity depend on theoretical context. Steel (1996); Myrvold (1996) provide such discussions within the Bayesian paradigm.

A major blow to the correlation approach was dealt by Bovens and Hartmann $(2002,2003)^{3}$. Their starting point is different, (Bovens and Hartmann, 2003, p. 93-94) "interpret more varied evidence as evidence that stems from multiple instruments (rather than a single instrument) and that tests multiple testable consequences (rather than a single testable consequence) of the hypothesis". Their analysis unearths cases in which their VET fails within their models of scientific inference.

Furthermore, they also show that if varied evidence is conceptualised in the correlational sense, then the principle that more varied evidence leads, ceteris paribus, to more confirmatory support to the hypothesis fails in particular circumstances (Bovens and Hartmann, 2003, p. 104-106). The counter-intuitive results obtained by Bovens and Hartmann do not contradict Earman's results since they relax the assumption that the hypothesis has to entail the evidence and they hence consider a much larger set of situations. The VET fails for some of these situations which were not considered by Earman (Bovens and Hartmann, 2003, Section 4.2 and 4.3).

The Bayesian quest for a vindication of the VET in full generality has failed. While the quest for a vindication of the VET is over, a case could be made

\footnotetext{
${ }^{3}$ Fresh interest in their model for their model in (the philosophy of) pharmacology is taken in Landes et al. (2017).
} 
for the VET in particular epistemological cases or applications. The only such attempt is that of Claveau (2013) in the philosophy of science.

His starting point is a different construal of the unreliability of scientific instruments. Rather than construing unreliable instruments as complete randomisers the approach taken by Bovens and Hartmann - he models unreliable instruments as systematically biased. His models satisfy a version of the VET on first pass, see (Claveau, 2013, Section 4). ${ }^{4}$ On second pass, Claveau drops the assumption that the systematic biases of instruments are fully independent. In this enlarged class, in which systematic biases of instruments are dependent to some degree, his version of the VET fails in certain cases, see (Claveau, 2013, Section 5). Seemingly puzzled and/or unsatisfied by his findings, (Claveau, 2013, p. 113) ends his discussion with: "The fate of the variety-of-evidence thesis is not yet settled".

Approaches to the VET are summarised in Table 1. Lacking in all these approaches is a widely-applicable explication of the notion of varied evidence. Neither Horwich nor his successors attempt an explication. Earman only considers the variety of a body of evidence which follows deductively from the hypothesis and the background knowledge. The only other ingredient to his explication of variety of evidence is the agent's prior. To us, this seems too meagre a recipe for a well-rounded explication. Bovens and Hartmann do not offer an explication. Claveau's explication only applies to bodies of evidence consisting of two items of evidence.

\begin{tabular}{|l|l|}
\hline Approach & Variety in terms of \\
\hline \hline Correlation & Correlation of items of evidence \\
\hline Eliminative & Disconfirmation of competing hypotheses \\
\hline $\begin{array}{l}\text { Sources of Evidence \& Con- } \\
\text { sequences of the Hypothesis }\end{array}$ & $\begin{array}{l}\text { Multiple consequences of the hypothesis } \\
\text { and/or multiple instruments }\end{array}$ \\
\hline
\end{tabular}

Table 1: Different approaches to the Variety of Evidence Thesis.

\subsection{The next Chapter}

Bayesians who hold the VET dear and are thus deeply troubled by the negative results obtained by Bovens and Hartmann and Claveau face the challenge of squar-

\footnotetext{
${ }^{4}$ This is consistent with the Bovens and Hartmann approach since their analysis "does not apply to unreliable instruments that do not randomize" (Bovens and Hartmann, 2003, p. 95).
} 
ing their bond to the VET and these negative results which spell an end to the unconditional love of the VET.

The plan for battle is a follows: First, we devise models scientific inference in which it makes sense to think about varied evidence. We put forward such models in Section 3 drawing on the Bovens and Hartmann approach. We then explicate a notion of varied evidence (Section 4) within our models. This puts us in a position to offer an explication of the VET within these models of scientific inference (Section 5). We argue in Section 5.3 that our model and explication are better suited for the philosophy of science than those of Bovens and Hartmann and Claveau. Furthermore, we argue that their models do not track how scientists think about the reliability of their instruments, conclusions drawn within their models are hence of limited interest in (the philosophy of) science. In Section 6, we show that the VET holds in all our models.

Interestingly, our models not only pronounce on confirmatory evidence but also on disconfirmatory and discordant evidence, see Theorems 4 and 5. Disconfirmation is rarely discussed in the Bayesian literature while featuring prominently in Popperian writings. Our models give the - we think - intuitively right result for disconfirmatory and discordant evidence, see Section 6.2.2 and Section 6.2.3.

We claim to establish that the VET can be given a formal justification within a variety of models of scientific inference and gesture at a re-examination of intuitions which drive the VET (Section 7).

The theoretical discussion is accompanied by an episode in the history of science in which variety of evidence clinched the argument: the thirteen ways of determining Avogardo's constant from the hypothesis that matter consists of molecules (atomism), see Perrin (1924). (Poincaré, 1963, p. 91) famously conceded his opposition to atomism when presented with a highly diverse body of evidence: "What makes it all the more convincing are the multiple correspondences between results obtained by totally different processes."

\section{Models of Scientific Inference}

\subsection{Variables}

We base our models on two widely-held beliefs. i) Scientific inference is amenable to Bayesian modelling and ii) concerned with the status of scientific hypotheses.

Our models of scientific inference use a binary propositional hypothesis variable, $H$, where $h$ stands for the proposition that the hypothesis of interest is true 
(e.g., 'matter consists of molecules'). ${ }^{5}$ The reader interested in the descriptive adequacy of a hypothesis may replace 'true' by 'descriptively adequate' and 'false' by 'not descriptively adequate' throughout.

For a body of evidence $\mathcal{E}$, we shall be interested in the Bayesian probability of $H$ being true, $P_{\mathcal{E}}(h)$. We freely admit that not all scientific inferences can be captured by our models, for example we only consider direct evidence for or against a hypothesis of interest; indirect evidence such as the No Alternative Argument of Dawid et al. (2015) is not considered here.

Next, we incorporate into our models that scientific hypotheses are typically not directly tested, rather it is some of their observable consequences which are testable (Bovens and Hartmann, 2003, p. 89). We shall use two sets of binary propositional consequence variables, the $C_{n}$ and the $D_{k}$. The values $c_{n}$, respectively, $d_{k}$, stand for the proposition that the testable consequence $C_{n}$, respectively, $D_{k}$, of $H$ holds. ${ }^{6}$ Evidence pertaining to the first set of variables will vary for evidential situations we compare, while the evidence pertaining to the $D_{k}$ variables will not vary. $\mathcal{C}$ denotes the set of all consequence variables.

In our running example for the hypothesis $H$ that 'matter consists of molecules', the testable consequences might be: i) 'the sky is blue' (Perrin, 1924, §83, pp. 197), ii) 'at room temperature, the viscosity of hydrogen is $0.88 \cdot 10^{-5} \mathrm{Nms}^{-2}$ ' (Perrin, 1924, $\S 46$, pp. 107), iii) 'the pressure in in an emulsion is exponentially decreasing with decreasing depth' (Perrin, 1924, §54, pp. 129), iv) 'black bodies emit electromagnetic radiation with the black body spectrum' (Perrin, 1924, §88-90, pp. 211).

Finally, measurements of a testable consequence are modelled by an evidential propositional variable. We use variables $E_{l}$ to represent evidence pertaining to the $C_{n}$. Furthermore, the evidence variables $F_{l}$ pertains to the $D_{k} . e_{l}$, respectively, $f_{l}$, stand for the proposition that a (series of) measurement(s) of the quantity of interest has resulted in some value. Let $e^{+}$stand for the proposition that this value is consistent with $C$ (e.g., 'the average measured quantity of helium produced by one gram of radium per day in our lab was $0.02 \mathrm{~mm}^{3}$ '). As usual, closes misses count as hits. What counts as a close in a particular application depends. Eviden-

\footnotetext{
${ }^{5}$ It is irrelevant for our discussion whether the hypothesis in question is deterministic or statistical, e.g., ' $85 \%$ of patients treated with drug $D$ recover more quickly than patients not receiving any treatment'.

${ }^{6}$ Variety of Evidence reasoning in climate science has recently been analysed to proceed via different consequences of the hypothesis that temperatures are rising, see Vezér (2017). Consequence considered were patterns in temperature profiles in ice, rock and soil as well as the lengths of mountain glaciers and sizes of tree rings.
} 
tial variables have finite arity which is obtained by suitably discretising the set of possible measurements.

For a variable $V$ we also let $v^{1}:=v$ and for the negation we put $v^{0}:=\bar{v}$.

\subsection{Topology of Bayesian Networks}

We use Bayesian networks to represent and reason with probability functions defined over the space spanned by these variables. The topology of the directed acyclic graphs of the Bayesian networks is adopted from Bovens and Hartmann (2002).

The hypothesis variable $H$ is the unique root node. The children of $H$ are the consequence variables in $\mathcal{C}$. They have, in turn, a number of evidential variables as children. ${ }^{7}$ Every child of a consequence variable has exactly one parent. There are no further edges in the Bayesian network. See Figures 1 and 2 for example networks. ${ }^{8}$ The assumed independences can be read off the Bayesian networks using the usual $d$-separation criterion, see for example Pearl (2009). Consequence variables without children represent lines of scientific inquiry which are currently unexplored.

This topology is generated by the following modelling choices regarding probabilistic independences and dependences. We first consider the independences, i.e., the missing edges. Firstly, $H$ screens off every consequence variable from every other consequence variable:

$$
C \perp C^{\prime} \mid H \text { for all different } C, C^{\prime} \in \mathcal{C} .
$$

For example, the probability that the colour of the sky is blue, $P(c)$, depends on whether atomism $(H)$ holds but does not also depend on the viscosity of hydrogen at room temperature $\left(C^{\prime}\right)$.

Furthermore, a testable consequence variable screens $C$ off its evidence from the hypothesis $H$

$$
E \perp H \mid C \text { for all } C \in \mathcal{C} \text { and all children } E \text { of } C .
$$

The probability that the colour of the sky is observed to be blue depends on whether the sky is blue; but this probability does not also depend on the truth of atomism, $P\left(e^{+} \mid c h\right)=P\left(e^{+} \mid c\right)$.

\footnotetext{
${ }^{7}$ As suggested as future work in (Bovens and Hartmann, 2002, p. 65, (II)), we consider consequence variables which may have three or more children.

${ }^{8}$ See Section 5.2 for a generalisation of the network topology.
} 
Finally, a consequence variable $C$ screens off its evidence variables

$$
E \perp E^{\prime} \mid C \text { for all } C \in \mathcal{C} \text { and all different children } E, E^{\prime} \text { of } C .
$$

This says that conditionalising on a consequence variable renders their children variables independent. ${ }^{9}$

We now turn to the edges present in our models. The probability of whether a testable consequence holds or not is directly influenced by whether the hypothesis of interest holds or not. Similarly, the probabilities of measurements coming out a certain way directly depends on whether the relevant testable consequence of the hypothesis holds. This motivates the edges and their orientation.

\subsection{Probabilities for Bayesian Networks}

To avoid unnecessary technical complications, we assume that all conditional probabilities in our Bayesian networks are non-zero.

That $C$ is a consequence of $H$ means that $C$ is probabilistically entailed by $H$ and hence that $C$ is more likely under $H$ than under its negation. We hence require (as do Bovens and Hartmann (2003)) that $P(c \mid h)>P(c \mid \bar{h})$ or equivalently

$$
P(\bar{c} \mid h)<P(\bar{c} \mid \bar{h}) .
$$

If $C$ were a deductive consequence of the hypothesis $H$, then $P(c \mid h)=1$ and hence $P(c \mid h)+P(c \mid \bar{h})=1+P(c \mid \bar{h})>1$ holds. We shall here relax the requirement that $C$ is a deductive consequence of $H$ and only require that $P(c \mid h)$ is so large that ${ }^{10}$

$$
P(c \mid h)+P(c \mid \bar{h})>1 .
$$

When is a measurement which is in agreement with the predicted theoretical value likely? Clearly, it is more likely if the theory used for predicting it is true than if this theory is false. In mathematical prose this is

$$
P\left(e^{+} \mid c\right)>P\left(e^{+} \mid \bar{c}\right) \text { for all } C \in \mathcal{C} \text { and all its children } E .
$$

\footnotetext{
${ }^{9}$ Evidence variables which are confirmationally independent regarding the hypothesis variable and their role in confirming the hypothesis with respect to different confirmation measures are investigated in Fitelson (2001).

${ }^{10}$ Bovens and Hartmann only require that $P(c \mid h)>P(c \mid \bar{h})$. None of their counter-intuitive cases in which the VET fails depend on whether $P(c \mid h)=1$. Claveau does not employ consequence variables for this very reason (Claveau, 2013, p. 96). For us, testable consequences are instrumental for explicating the notion of varied evidence, see Section 4, and are hence part of our models.
} 
This is not a condition with sharp teeth: $P\left(e^{+} \mid c\right)$ is close to one, it is a Bayesian agent's degree of belief that, if the testable consequence $C$ holds, the experiment turns out as predicted. $P\left(e^{+} \mid \bar{c}\right)$ will be close to zero: if the testable consequence fails to hold, then there is a wide range of possibilities the experiment could turn out. Hence, every particular result of the experiment, here $e^{+}$, is unlikely. So, $P\left(e^{+} \mid \bar{c}\right)$ is close to zero. It follows that

$$
1 \gtrsim P\left(e^{+} \mid c\right) \gg P\left(e^{+} \mid \bar{c}\right) \gtrsim 0 \text { for all } C \in \mathcal{C} \text { and all its children } E \text {. }
$$

Modelling assumptions are discussed in Section 7.

\section{Variety}

\subsection{Varieties of Evidence}

While Bovens and Hartmann (2003) and Claveau (2013) are interested in a variety of sources of evidence, we are interested in how varied a body of evidence is with respect to the hypothesis of interest. Background information about the sources of evidence is here taken care of by the all important prior probability distribution. We can think of two natural ways in which bodies of evidence formalised within the present models may vary thusly. Firstly and more importantly, the items in a body of evidence may pertain in different ways to different consequences of the hypothesis. This sort of variety is thus fully determined by the topology of the Bayesian network and is pursued here. The idea to understand variety in terms of the topology of the Bayesian network is not new, it is already in Bovens and Hartmann (2002, 2003).

Secondly, the items in a body of evidence may be informative to different degrees about consequences of the hypothesis. This second sort of variety depends on actual conditional probabilities, which is left to further study. Clearly, two bodies of evidence formalised within the present models may vary in both these senses at the same time and in further senses.

Those who prefer science to be as objective as possible might appreciate that our topological explication is based on facts of the matter: which item pertains to which consequence. The choice of a prior - consistent with the topology - does not affect the degree of variety. This can be seen as an answer to (Wayne, 1995, pp. 115-116) who claimed that Bayesians had to give a satisfactory account of their judgement of diversity. 
Our explication of the notion of varied evidence is but one way to formalise some intuitions regarding varied evidence. We side with (Howson and Urbach, 2006, p. 125) in doubting that the grand project of giving one explication which captures all senses of varied evidence can ever successfully be completed since our intuitions seem too vague.

\subsection{Explicating Varied Evidence}

A variety of objects is a set of objects in which few (very few or even no) objects look the same. Clearly, variety is the sort of thing which comes in degrees. A degree of variety then measures how different objects are in some fixed set of objects. A degree of variety thus measures the degree of dis-order or chaos in some set. The most widely used measure of (dis-)order in (the philosophy of) the sciences is Shannon Entropy, see Shannon (1948). ${ }^{11}$

With this understanding of what 'variety of evidence' may mean and with models of scientific inference in place we now proceed to explicate the degree of variety of a body of evidence.

We use $|\mathcal{E}|$ to denote the total number of items of evidence in $\mathcal{E}$ and $|C|$ for the number of children of consequence variable $C$. The fraction $\frac{|C|}{|\mathcal{E}|}$ then gives the proportion of items of evidence which are children of $C$. The variety $V$ of a body of evidence $\mathcal{E}$ can then be defined by ${ }^{12}$

$$
V(\mathcal{E}):=-\sum_{C \in \mathcal{C}} \frac{|C|}{|\mathcal{E}|} \cdot \log \left(\frac{|C|}{|\mathcal{E}|}\right) .
$$

This is in close analogy with Shannon Entropy for probability functions $w$ defined on some finite not-empty set of possible worlds $\Omega$ by

$$
H(w):=-\sum_{\omega \in \Omega} w(\omega) \cdot \log (w(\omega))
$$

Our explication of variety formalises the degree of uniformity with which the items of evidence pertain to a variety of testable consequences of the hypothesis.

\footnotetext{
${ }^{11} \mathrm{~A}$ variety of measures of entropy have been put forward. The well of measures of entropy shows no sign of drying up any time soon, see Csiszár (2008) for an overview of entropies and their axiomatic characterisations.

${ }^{12}$ Adopting the standard convention that $0 \cdot \log (0):=0$.
} 


\subsection{Shannon's Axioms for Explicating Varied Evidence}

Shannon's axiom $H 1$ says that the measure of entropy should be continuous in the probabilities $w(\omega)$. In our discrete application (a finite number of items of evidence are children of a finite number of consequences of the hypothesis), this amounts to the requirement that the variety of a large body of evidence varies only little, if one item of evidence is adopted by a new parent. This strikes us reasonable, small changes in the topology of the Bayesian network entail only small changes in the variety of evidence. Our measure $V$ tracks this thought.

The degree of variety $V(\mathcal{E})$ increases with increasingly larger bodies of evidence in which every consequence variable has exactly one child. This is (Shannon, 1948, Section 6)'s axiom H2. This seems right, the variety of a body of evidence which has maximal variety (no two evidence variables look the same [have the same parent]) increases with the size of the body of evidence.

Shannon's third and final axiom H3 formalises "conditional entropy". Conditionals have long interested philosophers and a number of competing analyses have been put forward. It is hence not surprising that different measures of entropy do not satisfy Shannon's axiom H3. In general, different measures of entropy disagree about which of two probability functions has greater entropy. For an illustration of different intuitively plausible measures of entropy disagreeing on which probability function has greater entropy see, e.g., (Landes and Williamson, 2013, Figure 1). For example, our variety measure (employing Shannon Entropy) pronounces on whether body of evidence $\mathcal{E}$ with $\left|C_{1}\right|=5,\left|C_{2}\right|=3,\left|C_{3}\right|=2,\left|C_{4}\right|=$ 2 or body of evidence $\mathcal{E}^{\prime}$ with $\left|C_{1}^{\prime}\right|=4,\left|C_{2}^{\prime}\right|=4,\left|C_{3}^{\prime}\right|=3,\left|C_{4}\right|=1$ has greater variety. ${ }^{13}$ An argument as to whether $\mathcal{E}$ or $\mathcal{E}^{\prime}$ has greater variety could be made either way: the evidence pertaining to $C_{1}$ and $C_{2}$ is more varied for $\mathcal{E}^{\prime}$ while the evidence pertaining to $C_{3}$ and $C_{4}$ is more varied for $\mathcal{E}$.

\subsection{Two Conditions: Novel Evidence and Adoption}

We next identify two conditions every entropy measure employed in an explication of varied evidence ought to satisfy. If one of these conditions is satisfied, then it is intuitive which of two bodies of evidence has greater evidential variety. We shall hence only consider ordered pairs of bodies of evidence which satisfy one of these conditions. There may be further conditions under which such comparisons are intuitive.

\footnotetext{
${ }^{13}$ For the record, $\mathcal{E}$ has a slightly greater variety score the $\mathcal{E}^{\prime}: V(\mathcal{E})=1.3086>1.2861=$ $V\left(\mathcal{E}^{\prime}\right)$.
} 
Condition A: The variety of a body of evidence $\mathcal{E}$ increases when a novel item of evidence is discovered which is the only-child of its parent consequence variable, see Figure 1 for a network representation. For this larger body of evidence $\mathcal{E}^{\prime}$, we have that $V(\mathcal{E})<V\left(\mathcal{E}^{\prime}\right)$ (Proposition 4).
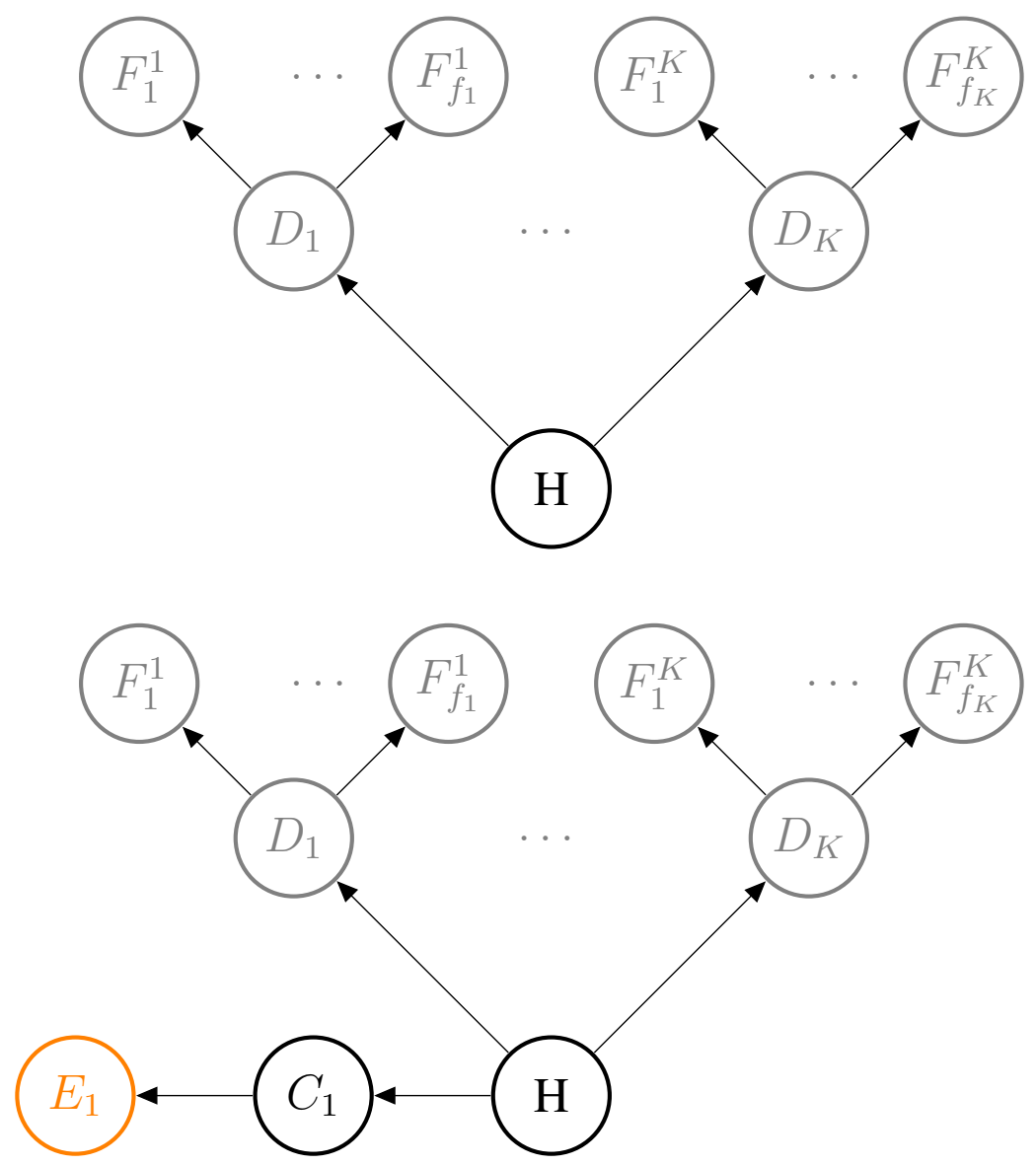

Figure 1: General Bayesian network model of Condition A in which a novel item of evidence has been added. It pertains to a consequence for which there was no evidence available.

Condition B: The variety of a body of evidence increases $\mathcal{E}$ when an item of evidence switches its parent variable from $C$ to $C^{\prime}$ such that, prior to the switch, $|C| \geq\left|C^{\prime}\right|+2$. Figuratively speaking: $C^{\prime}$ adopts a child of $C$ such that $C^{\prime}$ ends up not having more children than $C$, see Figure 2 for graphical illustration. This is 
intuitively right, the more evenly spread out the items of evidence are, the greater the variety of evidence, ceteris paribus.

Denoting the body of evidence after the adoption by $\mathcal{E}^{\prime}$, we now show that $V(\mathcal{E})<V\left(\mathcal{E}^{\prime}\right)$. Shannon Entropy strictly increases when the probability of some $\omega \in \Omega$ is reduced by $\alpha \in(0,1)$ and added to $w(\nu)$ some $\nu \in \Omega$, if $w(\omega)-\alpha>$ $w(\nu)$. This follows immediately from the strict concavity of $H$. We can conclude that $V(\mathcal{E})<V\left(\mathcal{E}^{\prime}\right)$.

Our "continuous" measure $V$ satisfies $\mathrm{H} 2$ and agrees with these two intuitive comparisons of evidential variety.

\subsection{The Non-Issue of Normalisation}

Shannon Entropy is defined for probability functions while our measure of variety $V(\mathcal{E})$ is defined on formal representations of bodies of evidence. To strengthen the analogy, we normalised by dividing by $|\mathcal{E}|$. Had we not normalised, we would have defined

$$
V_{1}(\mathcal{E}):=-\sum_{C \in \mathcal{C}}|C| \cdot \log (|C|)
$$

Since we are not interested in the absolute variety of a body of evidence but rather in which body of evidence has the comparatively greater variety, the following proposition shows that normalising is unproblematic for bodies of evidence of equal size:

Proposition 1. For bodies of evidence $\mathcal{E}, \mathcal{E}^{\prime}$ with $|\mathcal{E}|=\left|\mathcal{E}^{\prime}\right|$ it holds that

$$
V(\mathcal{E}) \geq V\left(\mathcal{E}^{\prime}\right), \quad \text { if and only if } \quad V_{1}(\mathcal{E}) \geq V_{1}\left(\mathcal{E}^{\prime}\right)
$$

Non-trivial proofs of main arguments, such as this one, can be found in Appendix A.

Whatever the technical details, the key idea is this: An entropy measure can be used to explicate the notion of varied evidence.

\section{Explicating the Variety of Evidence Thesis}

With an explication of varied evidence in place, we can now proceed to offer an explication of the VET. 


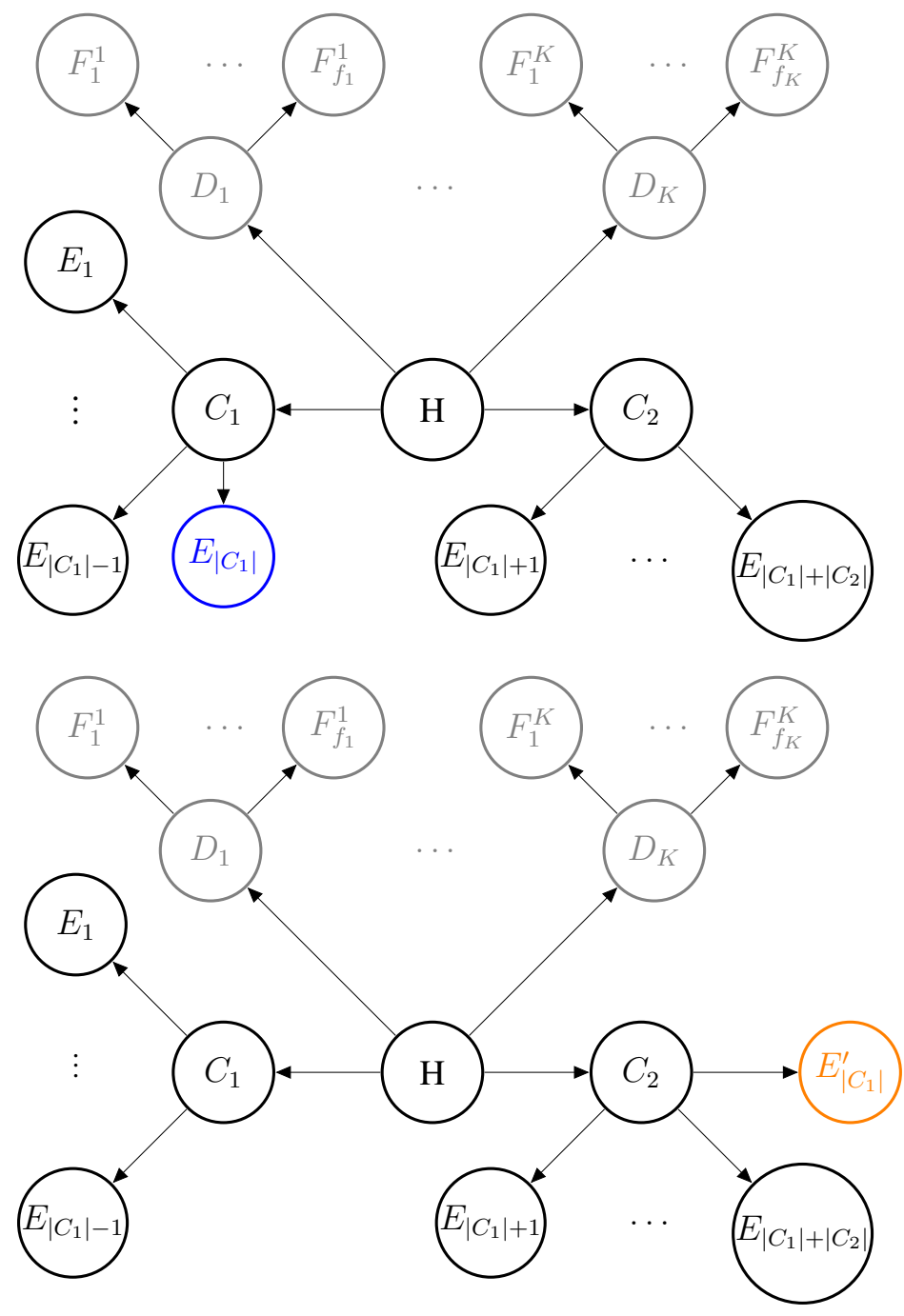

Figure 2: General Bayesian network model of Condition $\mathrm{B}$ where $E_{\left|C_{1}\right|}$ has switched parents and become a child of the consequence variable $C_{2}$. Again, the evidence pertaining to the $D$ variables remains stable and is shaded grey. The switching variable is highlighted in blue (prior to the switch) and orange (after the switch).

\subsection{Our Variety of Evidence Thesis}

We follow (Claveau, 2013, p. 95) and put forward our 
Variety of Evidence Thesis. Ceteris paribus, the strength of confirmation of a hypothesis by an evidential set of independent and confirmatory items of evidence increases with the diversity of the evidential elements in that set.

which we shall explicate in our models of scientific inference. We added the independence clause and the condition concerning confirmatory evidence which we will discuss in Section 6.2.3 and Section 7. Here, we shall only consider two Scenarios.

In Scenario A, two bodies of evidence $\mathcal{E}, \mathcal{E}^{\prime}$ satisfy Condition A; in Scenario $B$ they satisfy Condition B. These are however not all the restrictions we impose. Ceteris paribus means all other things being equal, respective conditional probabilities included. In both scenarios, (conditional) probabilities of the same variables remain unchanged. In Scenario B, this means that the conditional probabilities of $E_{\left|C_{1}\right|}$ given $C_{1}$ have to equal those $E_{\left|C_{1}\right|}^{\prime}$ given $C_{2}$, the conditional probabilities of $C_{1}$ given $H$ have to equal those of $C_{2}$ given $H$ [Ceteris Paribus] ${ }^{14}$ and finally the set of children of $C_{2}$ has to be equally confirmatory as a sub-set of children of $C_{1}$ [Pairing Off], see Table 2.

Finally, we need to require that evidence is confirmatory, see Table 2. To do so, we simplify notation by abbreviating the conditional probabilities of evidence pertaining to $C_{1}, C_{2}$ as follows:

$$
\chi_{1 s}:=\prod_{n=1}^{\left|C_{1}\right|-1} P\left(e_{n} \mid c_{1}^{s}\right) \quad \text { and } \quad \chi_{2 s}:=\prod_{g=1}^{\left|C_{2}\right|} P\left(e_{\left|C_{1}\right|+g} \mid c_{2}^{s}\right)
$$

We are ready to state our

\section{Explication of the Variety of Evidence Thesis}

In case of Scenario A or Scenario B it holds that $P_{\mathcal{E}}(h)<P_{\mathcal{E}^{\prime}}(h)$.

Note that we do not require $E_{\left|C_{1}\right|}$ and $E_{\left|C_{1}\right|}^{\prime}$ to have the same arity. We are thus only establish results within the models which concerns the observed evidence. Results for all possible observations are established by requiring the conditions for all truth values $e_{\left|C_{1}\right|}, e_{\left|C_{1}\right|}^{\prime}$ of $E_{\left|C_{1}\right|}, E_{\left|C_{1}\right|}^{\prime}$. Doing so, one restricts attention to cases in which $E_{\left|C_{1}\right|}, E_{\left|C_{1}\right|}^{\prime}$ do have the same arity.

\footnotetext{
${ }^{14}$ The case for treating two observable consequence on equal epistemological footing in (the reconstruction of) scientific inference has recently been defended in Parkkinen (2016).
} 


\begin{tabular}{|c|c|}
\hline Network Topology & Condition A \\
\hline Confirmatory Evidence & $P\left(e_{1} \mid c_{1}\right)>P\left(e_{1} \mid \bar{c}_{1}\right)$ \\
\hline Network Topology & Condition B \\
\hline \multirow{3}{*}{ Confirmatory Evidence } & $P\left(e_{\left|C_{1}\right|} \mid c_{1}\right)>P\left(e_{\left|C_{1}\right|} \mid \bar{c}_{1}\right)$ \\
& $\prod_{l=\left|C_{2}\right|+1}^{\left|C_{1}\right|-1} P\left(e_{l} \mid c_{1}\right)>\prod_{l=\left|C_{2}\right|+1}^{\left|C_{1}\right|-1} P\left(e_{l} \mid \bar{c}_{1}\right)$ \\
\hline & $\chi_{11} \cdot \chi_{21} \geq \chi_{10} \cdot \chi_{20}$ \\
\hline Ceteris Paribus & $P\left(e_{\left|C_{1}\right|} \mid c_{1}\right)=P\left(e_{\left|C_{1}\right|}^{\prime} \mid c_{2}\right)$ \\
& $P\left(e_{\left|C_{1}\right|} \mid \bar{c}_{1}\right)=P\left(e_{\left|C_{1}\right|}^{\prime} \mid \bar{c}_{2}\right)$ \\
& $P\left(c_{1} \mid h\right)=P\left(c_{2} \mid h\right)$ \\
& $P\left(c_{1} \mid \bar{h}\right)=P\left(c_{2} \mid \bar{h}\right)$ \\
\hline Paring Off & $\prod_{n=1}^{\left|C_{2}\right|} \frac{P\left(e_{n} \mid c_{1}\right)}{P\left(e_{n} \mid \bar{c}_{1}\right)}=\frac{\chi_{21}}{\chi_{20}}$ \\
\hline
\end{tabular}

Table 2: Scenario A (top) and Scenario B (bottom) under which more varied evidence ought to be more confirmatory.

The strict inequalities formalise that items of evidence have to be confirmatory, these constraints are rather weak. In Secnario B, we only require that the adopted item of evidence is somewhat confirmatory, as is the body of evidence which is not paired off. Furthermore, we require that the entire body of evidence without the adopted evidence variable is - as a whole - confirmatory. In particular, there is no requirement on the bodies of evidence to be strongly confirmatory in any sense; nor is there such a requirement on any particular item of evidence. Some items of evidence may even be disconfirmatory and the entire body of evidence may be discordant.

Considering Scenario A to be vacuous on the grounds that more evidence is always more confirmatory seems unduly hasty. It is well-known since (Carnap, 1962, p. 382) that a body of evidence consisting of two items of evidence, which both confirm the hypothesis of interest, may disconfirm the hypothesis. So, simply adding "confirmatory" item of evidence to an existing body of evidence does not always boost confirmation. Scenario A is also of interest as it concerns an increase in variety of evidence by the introduction of unexplored lines of inquiry along Shannon's axiom $\mathrm{H} 2$. 


\subsection{Alternative Explications}

We are interested in which of two bodies of evidence is more confirmatory. Hence, we are interested in which of the posterior probabilities $P_{\mathcal{E}}(h), P_{\mathcal{E}^{\prime}}(h)$ is greater. Our choice of confirmation measure is thus the difference of two posterior probabilities. Those interested in other confirmation measures in this context are referred to (Bovens and Hartmann, 2002, Appendix M) for discussion. Trivially, every choice of a confirmation measure which is ordinally equivalent to the difference measure yields same the comparisons of posterior probabilities. Thus, it delivers the same verdict about the VET.

One could also allow edges between the $D_{k}$ or allow the children of the $D_{k}$ to have multiple parents among the $D_{k}$. However, this renders the defined measure $V(\mathcal{E})$ meaningless since it ought to matter how exactly the evidence variables pertain to their parents. While the measure $V(\cdot)$ is meaningless, one can still talk about the variety $V$ of the restrictions of $\mathcal{E}, \mathcal{E}^{\prime}$ to the consequence variables where they disagree $\left(C_{1}, \ldots, C_{N}\right)$. If one thusly defines comparative degrees of variety, then all results derived here hold, too.

Alternatively, one could define a measure of variety $V^{\prime}$ under which the variety of the evidence pertaining to the $C_{n}$ is additive, i.e., $V^{\prime}(\mathcal{E})=V^{\prime}\left(\mathcal{E}_{D}\right)+V^{\prime}\left(\mathcal{E}_{C}\right)$ where the index denotes the restriction to these variables. Again, the obtained results hold in this alternative approach, too.

In the same vein, one can allow for consequence variables $D_{k}$ which have arbitrary infinite arity and our results still hold - still assuming non-zero conditional probabilities.

Another generalisation, is to employ a hypothesis variable with countable (finite or infinite) arity and consequence variables which all have the same countable (finite or infinite) arity. We have so far only be interested in a binary hypothesis variable and binary consequence variables. We require that the consequence variables have equal arity to ensure that our explication of variety makes sense for these more general models. Given - we think - natural ceteris paribus conditions, the established technical results also hold for these more general models as we show in Appendix B.

\subsection{Comparison to Bovens and Hartmann and Claveau}

In the approaches of Bovens and Hartmann and Claveau, bodies of evidence vary by the agent's judgements of the dependence of the sources which provided the evidence. Imposing ceteris paribus conditions, they study the confirmation pro- 
vided by different bodies of evidence under different (in)dependence assumptions. The key idea is that the more independent the sources the greater their notion of varied evidence. With thusly understood variety, they discover cases in which less varied bodies of evidence confirm the hypothesis more strongly than more varied bodies of evidence.

Since the main contributions of these works is to show that there are cases in which purportedly varied evidence confirms less strongly than less varied evidence, failing to offer an explication of variety is, in our view, not the main issue with these arguments. It seems that some explication of their notion of variety should be possible. We feel that the main problems with their arguments lie elsewhere, their use and construal of the notion of reliability of scientific instruments.

We avoid the notion of reliability by considering variety of evidence with respect to the hypothesis. Conceptually, we exclude reliability from our analysis of the VET since the notion of reliability is - neither directly nor indirectly - invoked in the VET. Our analysis is thus closer in spirit to the epistemological thesis under investigation. Reliability is subsumed here in the all-important Bayesian prior.

(Bovens and Hartmann, 2002, p. 33) model scientific instruments as either being fully reliable or as being a complete randomiser: either a scientific instrument distinguishes correctly whether the pertinent testable consequence $C$ holds [fully reliable] or it reports the value predicted by the pertinent testable consequence $C$ with fixed probability $a$, regardless of whether $C$ holds or not [complete randomiser]. In the latter case, all measurements from this instrument provide no information whatsoever.

We agree with (Claveau, 2013, p. 96) "The unreliable sources in their model are not like unreliable sources in actual science (i.e., their unreliable sources are randomly biased while systematic bias is far more likely to be the issue)". There is another problem with modelling scientific instruments as randomisers. Two conflicting measurements from the same instrument force us to conclude that the instrument is a randomiser. This seems much too strong.

While Claveau's model of (un)reliability is an improvement, it suffers from a similar problem. It is inconsistent with the agent's beliefs that the readings of an instrument on two runs of the same experiment differ, see (Claveau, 2013, p. 105). This cannot be right. When repeating a scientific experiment, it is normally very hard to guarantee that the (exact) initial and boundary conditions hold on multiple occasions. One should hence expect that the measurements disagree (to some degree) without suspecting a problem with measuring instruments. It hence seems, to us, that Claveau's formalisation of (un)reliability of scientific instruments does not adequately capture our intuitive understanding of (un)reliable scientific instru- 
ments. $^{15}$

\subsection{Comparison to Correlational and Eliminative Approach}

Our approach extends results in Earman (1992) since we consider bodies evidence which may not be entailed by the hypothesis, some items of evidence may even be inconsistent with the hypothesis.

There do not seem to be deep connections to the eliminative approach, in general. For two competing (mutually exclusive and jointly exhaustive) hypothesis $(H, \bar{H})$ we readily find for their posterior probabilities $P_{\mathcal{E}}(h)+P_{\mathcal{E}}(\bar{h})=1$. Hence, evidence which confirms $H$ more strongly is better at disconfirming the single competing hypothesis. ${ }^{16}$

With the generalisation to higher arity variables, our approach may be seen as an eliminative approach, see Appendix B. The hypothesis that $H$ is true is confirmed by evidence, if and only if the disjunction of all competing hypotheses is dis-confirmed by the evidence. We leave it to the reader to judge whether this does justice to the spirit of the eliminative approach.

\section{The Variety of Evidence Thesis}

We now discuss the status of the VET in our models.

\subsection{Novel Confirmatory Evidence}

We consider a fixed body of evidence $\mathcal{E}$ and add a new item of evidence which pertains to a consequence $C$ for which there is no other evidence available. The variety of this new body of evidence $\mathcal{E}^{\prime}$ has increased (Proposition 4 ). The posterior probability of $h$ increases, if the added new item of evidence is in agreement with its predicted value:

Theorem 1. In case of Condition A

$$
\operatorname{sign}\left(P_{\mathcal{E}}(h)-P_{\mathcal{E}^{\prime}}(h)\right)=\operatorname{sign}\left(P\left(e_{1} \mid \bar{c}_{1}\right)-P\left(e_{1} \mid c_{1}\right)\right) .
$$

\footnotetext{
${ }^{15}$ (Claveau, 2013, p. 113) offers another caveat of his results: "One could thus read the result as highlighting the danger of using extremely weak evidential sources, rather than as a direct refutation of the variety-of-evidence thesis."

${ }^{16}$ A rare meeting of Bayes and Popper, see also Section 6.2.2 for disconfirmatory evidence.
} 
Put differently

Corollary 1 (The VET - Scenario A). Condition $A$ and $P\left(e_{1} \mid c_{1}\right)>P\left(e_{1} \mid \bar{c}_{1}\right)$ imply

$$
P_{\mathcal{E}}(h)<P_{\mathcal{E}^{\prime}}(h)
$$

In other words, the more independent items of evidence which are in agreement with their respective predicted value, the greater the posterior probability of the hypothesis.

\subsection{Adoption}

As explained in Section 4.2, the variety of a body of evidence increases, when an item of evidence changes parents where the new parent has - after the switch - not more children than the previous parent, Condition B. The Bayesian networks for the corresponding bodies of evidence $\mathcal{E}, \mathcal{E}^{\prime}$ are depicted in Figure 2, with $\left|C_{1}\right| \geq$ $\left|C_{2}\right|+2$. For the remainder of this section, $\mathcal{E}, \mathcal{E}^{\prime}$ will denote an ordered pair of such bodies of evidence.

We obtain a surprisingly simple formula for the sign of $P_{\mathcal{E}}(h)-P_{\mathcal{E}^{\prime}}(h)$ :

Theorem 2. In case Condition B, the Ceteris Paribus Conditions and the Pairing Off Condition condition all hold, then

$$
\begin{aligned}
& \operatorname{sign}\left(P_{\mathcal{E}}(h)-P_{\mathcal{E}^{\prime}}(h)\right) \\
= & \operatorname{sign}\left(\left[P\left(e_{\left|C_{1}\right|} \mid \bar{c}_{1}\right)-P\left(e_{\left|C_{1}\right|} \mid c_{1}\right)\right] \cdot\left(\prod_{l=\left|C_{2}\right|+1}^{\left|C_{1}\right|-1} P\left(e_{l} \mid c_{1}\right)-\prod_{l=\left|C_{2}\right|+1}^{\left|C_{1}\right|-1} P\left(e_{l} \mid \bar{c}_{1}\right)\right)\right) .
\end{aligned}
$$

We note, all terms using the extra evidence $\vec{f}$, the paired-off evidence, the prior probability of $h$ and the conditional probabilities of the $C_{i}$ given $H$ have disappeared. Hence, the fate of the VET only depends on conditional probabilities of the non-paired-off evidence given (the negation of) testable consequences.

We now consider different evidential situations. First, we see what happens, if this evidence is in agreement with the predicted value. Then, we consider items of evidence which are all not in agreement with the predicted value. Finally, we consider discordant items of evidence. 


\subsubsection{Confirmatory Evidence}

Turning to confirmatory evidence, we find by applying Theorem 2 that for evidence which is more probable under a consequence $C$ than under its negations that:

Theorem 3 (The VET - Scenario B). If Condition B, the Ceteris Paribus Conditions and the Pairing Off Condition condition all hold and if for all $l \in$ $\left\{\left|C_{2}\right|+1, \ldots,\left|C_{1}\right|\right\}$ it holds that $P\left(e_{l} \mid c_{1}\right)>P\left(e_{l} \mid \bar{c}_{1}\right)$, then

$$
P_{\mathcal{E}}(h)<P_{\mathcal{E}^{\prime}}(h) .
$$

We now discuss the plausibility of our main results for confirmatory evidence, Corollary 1 and Theorem 3. Let us assume that we have conducted a number of independent experiments to measure the viscosity of hydrogen at room temperature $\left(C_{1}\right)$ and have not investigated any other consequence of $H$ ('matter consists of molecules'). All measurements were (around) $0.88 \cdot 10^{-5} \mathrm{Nms}^{-2}$. Our degree of belief in the hypothesis $H$ being true will take some value $d$. Further experiments regarding the viscosity of hydrogen at room temperature which agree with the predicted value will do little to change our belief in $H$ being true, even if these experiments are carried out at different places, at different times, by different people with different equipments.

Our models indeed track this line of thought that there is an upper bound to confirmation by only investigating a single consequence - even when all experiments turn out as predicted:

Corollary 2. If $E_{1}, \ldots, E_{\left|C_{1}\right|}$ are the children of $C_{1}$, then for all possible measurements $E_{1}=e_{1}, \ldots, E_{\left|C_{1}\right|}=e_{\left|C_{1}\right|}$

$$
P\left(h\left|e_{1} \ldots e_{\left|C_{1}\right|}\right| \vec{f}\right)<P\left(h \mid c_{1} \vec{f}\right)=\frac{1}{1+\frac{P(\bar{h}) \cdot P\left(c_{1} \mid \vec{h}\right) \cdot P(\vec{f} \bar{h})}{P(h) \cdot P\left(c_{1} \mid h\right) \cdot P(\vec{f} \mid h)}}<1 .
$$

The upper bound for the confirmation is smaller the further $C_{1}$ is from being a perfect indicator from $H$, i.e., the greater $P\left(c_{1} \mid \bar{h}\right)$ and the smaller $P\left(c_{1} \mid h\right)$.

Instead of conducting yet another experiment on the viscosity of hydrogen let us contemplate conducting an experiment on the radiation emitted by a black body $\left(C_{2}\right)$. If we detect the predicted black body radiation, then we are confident - to some degree - that black bodies do emit black body radiation. We hence become more confident that the consequences of $H$ are borne out across a variety 
of phenomena. Our degree of belief in the hypothesis $H$ being true increases non-negligibly beyond our prior degree of belief $d$.

This thinking is captured by Theorem 3 for the situation when there is no further background evidence, i.e., $\left|C_{2}\right|=0$ and $\vec{f}=\emptyset$. Corollary 1 and Theorem 3 demonstrate that our Bayesian models capture this reasoning in a wider range of circumstances, e.g., when there is further background knowledge $(\vec{f} \neq \emptyset)$ and/or a few experiments regarding the black body radiation had measured results in agreement with the predicted spectrum $\left(\left|C_{2}\right|>0\right)$.

In fact, we can calculate when it pays more to stop investigating the viscosity of hydrogen and move to investigating black body radiation. To simplify calculations we shall assume that $P\left(c_{1} \mid h\right)=1=P\left(c_{2} \mid h\right)$.

Proposition 2. If Condition B, the last two ceteris paribus condition and if $P\left(c_{1} \mid h\right)=1=P\left(c_{2} \mid h\right)$ hold, then

$$
\begin{aligned}
\operatorname{sign}\left(P_{\mathcal{E}}(h)-P_{\mathcal{E}^{\prime}}(h)\right)= & \operatorname{sign}\left(\frac{\sum_{j, l=0}^{1} P\left(c_{1}^{j} \mid \bar{h}\right) P\left(c_{2}^{l} \mid \bar{h}\right) P\left(e_{\left|C_{1}\right|}^{\prime} \mid c_{2}^{l}\right) \cdot \chi_{1 j} \cdot \chi_{2 l}}{P\left(e_{\mid C_{1}}^{\prime} \mid c_{2}\right)}\right. \\
& \left.-\frac{\sum_{j, l=0}^{1} P\left(c_{1}^{j} \mid \bar{h}\right) P\left(c_{2}^{l} \mid \bar{h}\right) P\left(e_{\left|C_{1}\right|} \mid c_{1}^{j}\right) \cdot \chi_{1 j} \cdot \chi_{2 l}}{P\left(e_{\left|C_{1}\right|} \mid c_{1}\right)}\right) .
\end{aligned}
$$

While this formula might appear somewhat opaque at first glance, we can already note that all the background $\vec{f}$ and the prior belief in $H$ have disappeared. In order to make progress, we need to have that the conditional probabilities of $E_{L+1} \mid C_{1}$ and $E_{L+1}^{\prime} \mid C_{2}$ are - at least for certain truth values - comparable. Making such an assumption we find

Proposition 3. If Condition $B$, the last two ceteris paribus condition, $P\left(c_{1} \mid h\right)=$ $1=P\left(c_{2} \mid h\right)$ and if $P\left(e_{\left|C_{1}\right|} \mid c_{1}\right)=P\left(e_{\left|C_{1}\right|}^{\prime} \mid c_{2}\right)>P\left(e_{\left|C_{1}\right|}^{\prime} \mid \bar{c}_{2}\right)$ hold, then

$$
\operatorname{sign}\left(P_{\mathcal{E}}(h)-P_{\mathcal{E}^{\prime}}(h)\right)=-\operatorname{sign}\left(\frac{\alpha_{0,0} \cdot \beta_{0,0}+\alpha_{0,1} \cdot \beta_{0,1}}{\alpha_{1,0} \cdot \beta_{1,0}}+\prod_{n=1}^{\left|C_{1}\right|-1} \frac{P\left(e_{n} \mid c_{1}\right)}{P\left(e_{n} \mid \bar{c}_{1}\right)}\right)
$$

where the $\alpha$ and $\beta$ are parameters independent of $\left|C_{1}\right|$ which are defined in (14).

In a given epistemic situation ( $\alpha$ and $\beta$ parameters and $\left|C_{2}\right| \geq 0$ fixed) there comes a point at which evidence in favor of black body radiation $\left(C_{2}\right)$ is more confirmatory than evidence in favor of the predicted viscosity of hydrogen $\left(C_{1}\right)$, the second summand is eventually greater than the first. For example, 
Corollary 3. Under the assumptions of Proposition 3, if there also exists some $\delta>0$ such that $\frac{P\left(e_{n} \mid c_{1}\right)}{P\left(e_{n} \mid \bar{c}_{1}\right)}>1+\delta=$ constant for all $n$, then for bodies of evidence $\mathcal{E}$ with

$$
\left|C_{1}\right| \geq 1+\log (1+\delta)-\log \left(\frac{\alpha_{1,0} \cdot \beta_{1,0}}{\alpha_{0,0} \cdot \beta_{0,0}+\alpha_{0,1} \cdot \beta_{0,1}}\right)
$$

it holds that

$$
P_{\mathcal{E}}(h)<P_{\mathcal{E}^{\prime}}(h) .
$$

It is worth pointing out that these results only require one weak ceteris paribus condition, $P\left(e_{\left|C_{1}\right|} \mid c_{1}\right)=P\left(e_{\left|C_{1}\right|}^{\prime} \mid c_{2}\right)$, the Pairing-Off Condition is not required.

The main point is this, there comes a point in life when investigating the exact same consequence yet again cannot provide significant further confirmation for the hypothesis of interest. Investigating a different consequence may be more confirmatory. A weaker version of this result is given in (Howson and Urbach, 2006, p. 95). Our argument is more general in that items of evidence are not necessarily logical consequences of the testable consequences, some items of evidence may even be strongly disconfirmatory in our result.

\subsubsection{Disconfirmatory Evidence}

Having discussed evidence confirming a hypothesis, we now turn to evidence which does not confirm to expectations. That is, we consider evidence which is more likely when consequences of the hypothesis are false.

Theorem 4. If Condition B, the Ceteris Paribus Conditions and the Pairing Off Condition conditions hold and if for all $l \in\left\{\left|C_{2}\right|+1, \ldots,\left|C_{1}\right|\right\}$ it holds that $P\left(e_{l} \mid c_{1}\right)<P\left(e_{l} \mid \bar{c}_{1}\right)$, then

$$
P_{\mathcal{E}}(h)<P_{\mathcal{E}^{\prime}}(h)
$$

Theorem 4 says that a more varied body of evidence which speaks against testable consequences entails a higher probability of $H$ being true than a less varied body of evidence. In other words, the probability of $H$ being true is lower, if evidence against the testable consequences is clustered around few or even just a single testable consequence.

Those interested in showing that a scientific hypothesis is (likely) false are thus interested in discovering disconfirmatory independent items of evidence which are concentrated on as few as possible different testable consequences, ceteris paribus. 
Let us consider whether this intuitively right. Assume for the moment that the $C_{n}$ are deductive consequences of the hypothesis. So, if only a single $C_{n}$ is known to be false, then we know that $H$ is false and assign zero posterior probability to $H$ being true.

In the running Perrin case, if the experiments conclusively rule out that black bodies emit black body radiation, then we can be sure that matter does not consist of molecules (holding the auxiliary background assumptions fixed as usual). A body of evidence which strongly discredits belief in black body radiation and in that the viscosity of hydrogen is $0.88 \cdot 10^{5} \mathrm{Nms}^{-2}$ at room temperature, makes for a (very) low, but non-zero, belief in atomism.

We can now use a continuity argument to pass from categorical ruling out of a consequence $C$ to evidence which strongly indicates (but not entails) the falsity of $C$ : a body of evidence strongly telling against one testable consequence $C$ is preferable for disconfirming the hypothesis to amassing a body of evidence which discredits the belief in the truth of multiple testable consequences, ceteris paribus. This thought is formalised in Theorem 4.

\subsubsection{Discordant Evidence}

There are a wide range of cases in which some of the evidence confirms a hypothesis while other evidence speaks against it. We here only consider cases in which one item of evidence, $E_{\left|C_{1}\right|}$, goes against the other items of evidence $E_{\left|C_{2}\right|+1}, \ldots, E_{\left|C_{1}\right|-1}$.

We obtain the following

Theorem 5. If Condition B, the Ceteris Paribus Conditions, the Pairing Off Condition conditions hold and if one of the following two conditions is satisfied

- $P\left(e_{l} \mid c_{1}\right)<P\left(e_{l} \mid \bar{c}_{1}\right)$ for all $l \in\left\{\left|C_{2}\right|+1, \ldots,\left|C_{1}\right|-1\right\}$ and $P\left(e_{\left|C_{1}\right|} \mid c_{1}\right)>$ $P\left(e_{\left|C_{1}\right|} \mid \bar{c}_{1}\right)$,

- $P\left(e_{l} \mid c_{1}\right)>P\left(e_{l} \mid \bar{c}_{1}\right)$ for all $l \in\left\{\left|C_{2}\right|+1, \ldots,\left|C_{1}\right|-1\right\}$ and $P\left(e_{\left|C_{1}\right|} \mid c_{1}\right)<$ $P\left(e_{\left|C_{1}\right|} \mid \bar{c}_{1}\right)$,

then

$$
P_{\mathcal{E}}(h)>P_{\mathcal{E}^{\prime}}(h)
$$

Theorem 5 expresses the thought that making the item of evidence which is discordant with other items of evidence more prominent by switching it to a new 
parent with fewer children makes for a comparatively lower posterior probability of $h$. Let us see whether this is right.

The theorem says that evidence in disagreement with one predicted value according to $C_{1}$ leads to a lower posterior probability of $h$, if the other evidence, which is in agreement with the predicted value, pertains to another testable consequence $\left(C_{2}\right)$, other things being equal. This is in line with our reasoning for the disconfirmation case. Disconfirmation is strongest, if disconfirmatory evidence is unopposed.

Let us recall (Claveau, 2013, p. 95)'s VET: "Ceteris paribus, the strength of confirmation of a hypothesis by an evidential set increases with the diversity of the evidential elements in that set." This version of the VET fails in our models for discordant evidence. As argued here, we think that it is right that more varied evidence confirms less strongly for discordant evidence. We suspect a slip in Claveau's writing. In the appendix in which Claveau proves his formal results, he only ever considers evidence which is in agreement with the predicted value. Furthermore, in (Claveau, 2011, p. 241) he writes "I take evidence to be always evidence for a specific proposition" [emphasis original]. He might have simply forgotten to state this convention in Claveau (2013). Bovens and Hartman and Earman also only consider confirmatory evidence.

We conclude our discussion of bodies of evidence (Sections 6.2.1-6.2.3) by stating the hope the reader agrees with us that our models also pronounce rightly on disconfirmation and discordant evidence.

\section{Discussion}

We now discuss two of our modelling assumptions (independent evidence and the novel condition 5) which are not in Bovens and Hartmann (2003) in two respects: i) their applicability in actual cases and ii) their adequacy for explicating the VET.

Addressing both assumptions and both respects we point out that idealisations made may only hold approximately in applications/may only be approximately adequate for explication of the VET. Since our main results (Corollary 1 and Theorem 3) are strict inequalities, these inequalities continue to hold in a neighbourhood of the idealisations, too. These neighbourhoods are the larger, the stricter the inequalities. In particular, for evidence which is in good agreement with the predicted value the inequality (6) is very strict. For such evidence, we hence expect the VET to be robust under non-drastic changes of idealisations made.

As for the applicability of the assumption of independent evidence we point to 
(Fitelson, 2001, Footnote 20) who defends an idealisation of independent evidence thusly: "I do not mean to suggest that confirmational independence can be used to undergird all of our intuitions about the value of diverse evidence. But, I do think that there are many important scientific cases that fit this mold" (our emphasis). We agree with these sentiments. However, when evidence is strongly dependent, for instance on sources, then neither our models nor our explication apply.

Concerning the adequacy of the independence assumption, we feel that it is mainly independence assumptions which drive the intuitive appeal of the VET. Again, we refer to (Fitelson, 2001, p. 131) who is thinking along similar lines: "I suspect that the notion of independent evidence can undergrid, at least partially, (some of) our intuitions about the significance of diverse evidence. At least one recent philosophers seems to share this suspicion. Sober (1989) shows [...]" (emphasis original). As we saw in Section 6.2.3, varied discordant evidence supports the hypothesis less than less varied evidence, ceteris paribus and under certain conditions. Out of this discussion a proponent of the VET might re-examine her intuitions and discover that they were mainly driven by an implicit assumption of independent evidence which supports the hypothesis. The intuition regarding dependent and/or discordant evidence may be felt less clearly or less strongly.

Strongly dependent evidence is another kettle of fish altogether. While we have not considered strongly dependent evidence here, we did cast some doubt on the applicability of the models by Bovens and Hartmann and Claveau for the philosophy of science, in which their formulation of the VET fails to hold for strongly dependent evidence. Clearly, there are many more ways (to formalise) in which evidence could be dependent - none of which we discussed here.

The condition that $P(c \mid h)$ is large is plausible in applications in science. After all, $C$ is a consequence of $H$. In our running example, the consequences are (almost) deductive consequence of the hypothesis. So, $P(c \mid h)$ is only marginally - if at all - less than one. Hence, any addition of a non-negligible amount of probability will make this sum larger than one, i.e., 5 holds. The same is true for the consequences considered in Landes et al. (2017); Vezér (2017). There are hence good reasons to think that 5 will hold in most applications in science.

The adequacy of condition 5 for the explication is less clear. While we feel that is reasonable to assume that 5 holds, others may disagree. They might point out that there is a symmetry between the hypothesis and its negation. Hence, if 5 were to hold, it would also need to hold for the negation of the hypothesis (due to a symmetry argument), too. This however entails a contradiction.

We think that such a symmetry is implausible since there is something special about scientific hypotheses and their consequences. Elementary logic tells us that 
if ' $H$ entails $C$ ', then it does not follow that ' $\bar{H}$ entails $\bar{C}$ '. For example, if matter would not consist of atoms, then it would not be a consequence that the sky is not blue. Likewise in (Landes et al., 2017, Figure 4), if a drug does not cause a side-effect, then it is not a consequence that there is no probabilistic dependence between drug use and observed side-effect; the observed side-effect may well be due to confounding. Scientific hypotheses tend to be specific and their testable consequences tend to be (almost) deductive consequences.

Nevertheless, it is a fact that when 5 fails, then there are instance in which our formulation of the VET fails for very specific parameter configurations for the adoption scenario. The VET then fails, if and only if ${ }^{17}$

$$
P\left(e_{\left|C_{1}\right|} \mid c\right) \cdot \alpha_{11} \cdot P(c \mid h) \cdot P(c \mid \bar{h})-P\left(e_{\left|C_{1}\right|} \mid \bar{c}\right) \cdot \alpha_{00} \cdot P(\bar{c} \mid h) \cdot P(\bar{c} \mid \bar{h})<0 .
$$

The evidence in the VET is taken to evidence for the hypothesis. It is hence very plausible that we are entitled to assume that $P\left(e_{\left|C_{1}\right|} \mid c\right)>P\left(e_{\left|C_{1}\right|} \mid \bar{c}\right)$ and that $\alpha_{11}>\alpha_{00}$. While the VET is silent on how strong the evidence confirms the conclusion (how strict these inequalities are), it seems safe to conclude that the VET only fails for very specific parameter values - when $P\left(e_{\left|C_{1}\right|} \mid c\right) \approx P\left(e_{\left|C_{1}\right|} \mid \bar{c}\right)$ and $\alpha_{11} \approx \alpha_{00}$. On the other hand, if 5 is accepted, then the VET does not fail.

Generally speaking, while these two modelling assumptions place further restrictions on the prior probability distribution our approach is more general than the Bovens and Hartmann model in that extends to variables of greater arity (Appendix B) and to bodies of evidence with three or more items of evidence.

\section{Conclusion}

Despite enjoying widespread intuitive support the VET has received bad press of late Bovens and Hartmann (2002, 2003); Claveau (2013); Fitelson (1996). A model-independent or explication-independent vindication of the VET is - given these negative results - a pipe-dream. Hence, at best one can hope for a wide class of pertinent models in which explications of the VET hold.

We here put forward an explication of the notion of varied evidence within a class of models of scientific inference by appealing to measures of entropy. Our explication of the VET holds in our models. Furthermore, our models also pronounce rightly on disconfirmation and discordant evidence, we think. This

\footnotetext{
${ }^{17}$ see (13) at the end of the proof of Theorem 2
} 
lends pertinence to the class of discussed models. We would hence argue that the case for the Variety of Evidence Thesis emerges strengthened.

It will be interesting to see, if the investigation of more realistic (read more complicated) models of scientific inference unearths more cases in which the VET fails or if the VET holds (much) more widely. One option is to consider measures of variety which take more senses of variety into account, such measures appear in the ecology literature on diversity, e.g., see Tuomisto (2010).

Much remains to be said about the Variety of Evidence Thesis.

Acknowledgements Blinded for review.

\section{References}

Borm, G. F., Lemmers, O., Fransen, J., and Donders, R. (2009). The evidence provided by a single trial is less reliable than its statistical analysis suggests. Journal of Clinical Epidemiology, 62(7):711-715.

Bovens, L. and Hartmann, S. (2002). Bayesian Networks and the Problem of Unreliable Instruments. Philosophy of Science, 69(1):29-72.

Bovens, L. and Hartmann, S. (2003). Bayesian Epistemology. Oxford University Press.

Carnap, R. (1962). Logical foundations of probability. University of Chicago Press, 2 edition.

Claveau, F. (2011). Evidential variety as a source of credibility for causal inference: beyond sharp designs and structural models. Journal of Economic Methodology, 18(3):233-253.

Claveau, F. (2013). The Independence Condition in the Variety-of-Evidence Thesis. Philosophy of Science, 80(1):94-118.

Csiszár, I. (2008). Axiomatic Characterizations of Information Measures. Entropy, 10(3):261-273.

Dawid, R., Hartmann, S., and Sprenger, J. (2015). The No Alternatives Argument. British Journal for the Philosophy of Science, 66(1):213-234.

Earman, J. (1992). Bayes or Bust? MIT Press. 
Fitelson, B. (1996). Wayne, Horwich, and Evidential Diversity. Philosophy of Science, 63(4):652-660.

Fitelson, B. (2001). A bayesian account of independent evidence with applications. Philosophy of Science, 68(3):S123-S140.

Franklin, A. and Howson, C. (1984). Why do scientists prefer to vary their experiments? Studies in History and Philosophy of Science Part A, 15(1):51-62.

Glymour, C. (1980). Theory and Evidence. Princeton University Press.

Hempel, C. (1966). Philosophy of Natural Science. Prentice Hall.

Horwich, P. (1982). Probability and Evidence. Cambridge University Press.

Horwich, P. (1998). Wittgensteinian Bayesianism. In Curd, M. and Cover, J. A., editors, Philosophy of Science: The Central Issues, pages 607-624. W. W. Norton \& Company.

Howson, C. and Urbach, P. (2006). Scientific Reasoning. Open Court, 3 edition.

Hüffmeier, J., Mazei, J., and Schultze, T. (2016). Reconceptualizing replication as a sequence of different studies: A replication typology. Journal of Experimental Social Psychology, 66:81-92.

Kenynes, J. M. (1921). A Treatise on Probability. MacMillan.

Landes, J., Osimani, B., and Poellinger, R. (2017). Epistemology of Causal Inference in Pharmacology. European Journal for Philosophy of Science. 47 pages.

Landes, J. and Williamson, J. (2013). Objective Bayesianism and the maximum entropy principle. Entropy, 15(9):3528-3591.

Lloyd, E. A. (2015). Model robustness as a confirmatory virtue: The case of climate science. Studies in History and Philosophy of Science Part A, 49:5868 .

Meehl, P. E. (1990). Appraising and Amending Theories: The Strategy of Lakatosian Defense and Two Principles that Warrant It. Psychological Inquiry, 1(2):108-141. 
Myrvold, W. C. (1996). Bayesianism and diverse evidence: A reply to Andrew Wayne. Philosophy of Science, 63(4):661.

Parkkinen, V.-P. (2016). Robustness and evidence of mechanisms in early experimental atherosclerosis research. Studies in History and Philosophy of Science Part C: Studies in History and Philosophy of Biological and Biomedical Sciences, 60:44-55.

Pearl, J. (2009). Causality Models, Reasoning and Inference. Cambridge University Press, 2 edition. Zbl 1188.68291.

Perrin, J. (1924). Les Atomes. Félix Alcan.

Poincaré, H. (1963). Mathematics and Science: Last Essays. Dover.

Schupbach, J. N. (2015). Robustness, Diversity of Evidence, and Probabilistic Independence, pages 305-316. Springer.

Schupbach, J. N. (2017). Robustness analysis as explanatory reasoning. British Journal for the Philosophy of Science. forthcoming.

Shannon, C. (1948). A Mathematical Theory of Communication. The Bell System Technical Journal, 27:379-423.

Steel, D. (1996). Bayesianism and the value of diverse evidence. Philosophy of Science, 63(4):666-674.

Tuomisto, H. (2010). A consistent terminology for quantifying species diversity? Yes, it does exist. Oecologia, 164(4):853-860.

Vezér, M. A. (2017). Variety-of-evidence reasoning about the distant past. European Journal for Philosophy of Science, 7(2):257-265.

Wayne, A. (1995). Bayesianism and diverse evidence. Philosophy of Science, 62(1):111-121. 


\section{A Proofs of Main Results}

We now give the longer proofs. The propositions to be proved are re-stated for convenience.

Proposition 1. For bodies of evidence $\mathcal{E}, \mathcal{E}^{\prime}$ with $|\mathcal{E}|=\left|\mathcal{E}^{\prime}\right|$ it holds that

$$
V(\mathcal{E}) \geq V\left(\mathcal{E}^{\prime}\right), \quad \text { if and only if } \quad V_{1}(\mathcal{E}) \geq V_{1}\left(\mathcal{E}^{\prime}\right)
$$

Proof. We begin by computing using that: $\sum_{C \in \mathcal{C}}|C|=|\mathcal{E}|$

$$
\begin{aligned}
V(\mathcal{E}) & =-\sum_{C \in \mathcal{C}} \frac{|C|}{|\mathcal{E}|} \cdot \log \left(\frac{|C|}{|\mathcal{E}|}\right) \\
& =-\frac{1}{|\mathcal{E}|} \cdot\left(\sum_{C \in \mathcal{C}}|C| \cdot \log \left(\frac{|C|}{|\mathcal{E}|}\right)\right) \\
& =-\frac{1}{|\mathcal{E}|} \cdot\left(\sum_{C \in \mathcal{C}}|C| \cdot(\log (|C|)-\log (|\mathcal{E}|))\right) \\
& =-\frac{1}{|\mathcal{E}|} \cdot\left(-V_{1}(\mathcal{E})+\sum_{C \in \mathcal{C}}|C| \cdot(-\log (|\mathcal{E}|))\right. \\
& =\frac{V_{1}(\mathcal{E})}{|\mathcal{E}|}+\log (|\mathcal{E}|) .
\end{aligned}
$$

Hence, $V_{1}(\mathcal{E})$ is a positive-slope affine-linear transformation of $V(\mathcal{E})$.

Proposition 4. For all $|\Omega| \geq 1$ and all $\vec{x}:=\langle w(\omega): \omega \in \Omega\rangle$ it holds that $H(\vec{x})<H\left(\frac{|\Omega|}{|\Omega|+1} \vec{x}, \frac{1}{|\Omega|+1}\right)$.

Proof. Using that $\sum_{i=1}^{|\Omega|} x_{i}=1$ and that $H(\vec{x}) \leq-\log \left(\frac{1}{|\Omega|}\right)=\log (|\Omega|)$ we find

$$
\begin{aligned}
& H\left(\frac{|\Omega|}{|\Omega|+1} \vec{x}, \frac{1}{|\Omega|+1}\right)-H(\vec{x}) \\
& \quad=-\frac{1}{|\Omega|+1}\left(\log \left(\frac{1}{|\Omega|+1}\right)+\sum_{i=1}^{|\Omega|}|\Omega| x_{i} \cdot \log \left(\frac{|\Omega|}{|\Omega|+1} x_{i}\right)\right)-H(\vec{x}) \\
& \quad=-\log \left(\frac{1}{|\Omega|+1}\right)-\frac{|\Omega|}{|\Omega|+1} \cdot\left(\sum_{i=1}^{|\Omega|} x_{i} \cdot\left[\log \left(x_{i}\right)+\log (|\Omega|)\right]\right)-H(\vec{x})
\end{aligned}
$$




$$
\begin{aligned}
& =\log (|\Omega|+1)-\frac{|\Omega|}{|\Omega|+1}(-H(\vec{x})+\log (|\Omega|))-H(\vec{x}) \\
& =\log (|\Omega|+1)-\frac{|\Omega|}{|\Omega|+1} \cdot \log (|\Omega|)-\frac{1}{|\Omega|+1} H(\vec{x}) \\
& \geq \log (|\Omega|+1)-\frac{|\Omega|}{|\Omega|+1} \log (|\Omega|)-\frac{1}{|\Omega|+1} \log (|\Omega|) \\
& =\log (|\Omega|+1)-\log (|\Omega|) \\
& >0
\end{aligned}
$$

Theorem 1. In case of Condition A

$$
\operatorname{sign}\left(P_{\mathcal{E}}(h)-P_{\mathcal{E}^{\prime}}(h)\right)=\operatorname{sign}\left(P\left(e_{1} \mid \bar{c}_{1}\right)-P\left(e_{1} \mid c_{1}\right)\right)
$$

Proof. To simply notation we let $\vec{f}$ denote the conjunction of all items evidence pertaining to the $D_{k}$ and obtain:

$$
\begin{gathered}
P_{\mathcal{E}}(h)=\frac{P(h \vec{f})}{P(\vec{f})}=\frac{P(\vec{f} \mid h) \cdot P(h)}{\sum_{s=0}^{1} P\left(\vec{f} \mid h^{s}\right) \cdot P\left(h^{s}\right)} \\
=\frac{1}{1+\frac{P(\vec{f} \mid \bar{h}) \cdot P(\bar{h})}{P(\vec{f} \mid h) \cdot P(h)}} \cdot \\
P_{\mathcal{E}^{\prime}}(h)=\frac{P\left(h e_{1} \vec{f}\right)}{P\left(e_{1} \vec{f}\right)}=\frac{P(\vec{f} \mid h) \cdot P(h) \cdot\left(\sum_{i=0}^{1} P\left(c_{1}^{i} \mid h\right) P\left(e_{1} \mid c_{1}^{i}\right)\right)}{\sum_{s=0}^{1} P\left(\vec{f} \mid h^{s}\right) \cdot P\left(h^{s}\right) \cdot\left(\sum_{i=0}^{1} P\left(c_{1}^{i} \mid h^{s}\right) P\left(e_{1} \mid c_{1}^{i}\right)\right)} \\
=\frac{1}{1+\frac{P(\vec{f} \mid \bar{h}) \cdot P(\bar{h}) \cdot\left(\sum_{i=0}^{1} P\left(c_{1}^{i} \mid \bar{h}\right) P\left(e_{1} \mid c_{1}^{i}\right)\right)}{P(\vec{f} \mid h) \cdot P(h) \cdot\left(\sum_{i=0}^{1} P\left(c_{1}^{i} \mid h\right) P\left(e_{1} \mid c_{1}^{i}\right)\right)}} .
\end{gathered}
$$

Hence,

$$
\begin{aligned}
& \operatorname{sign}\left(P_{\mathcal{E}}(h)-P_{\mathcal{E}^{\prime}}(h)\right) \\
= & \operatorname{sign}\left(\frac{1}{1+\frac{P(\vec{f} \mid \bar{h}) \cdot P(\bar{h})}{P(\bar{f} \mid h) \cdot P(h)}}-\frac{1}{1+\frac{P(\vec{f} \mid \bar{h}) \cdot P(\bar{h}) \cdot\left(\sum_{i=0}^{1} P\left(c_{1}^{i} \mid \bar{h}\right) P\left(e_{1} \mid c_{1}^{i}\right)\right)}{P(\bar{f} \mid h) \cdot P(h) \cdot\left(\sum_{i=0}^{1} P\left(c_{1}^{i} \mid h\right) P\left(e_{1} \mid c_{1}^{i}\right)\right)}}\right) \\
= & \operatorname{sign}\left(\frac{\sum_{i=0}^{1} P\left(c_{1}^{i} \mid \bar{h}\right) P\left(e_{1} \mid c_{1}^{i}\right)}{\sum_{i=0}^{1} P\left(c_{1}^{i} \mid h\right) P\left(e_{1} \mid c_{1}^{i}\right)}-1\right)
\end{aligned}
$$




$$
\begin{aligned}
& =\operatorname{sign}\left(\sum_{i=0}^{1} P\left(c_{1}^{i} \mid \bar{h}\right) P\left(e_{1} \mid c_{1}^{i}\right)-\sum_{i=0}^{1} P\left(c_{1}^{i} \mid h\right) P\left(e_{1} \mid c_{1}^{i}\right)\right) \\
& =\operatorname{sign}\left(P\left(e_{1} \mid c_{1}\right) \cdot\left[P\left(c_{1} \mid \bar{h}\right)-P\left(c_{1} \mid h\right)\right]+P\left(e_{1} \mid \bar{c}_{1}\right) \cdot\left[P\left(\bar{c}_{1} \mid \bar{h}\right)-P\left(\bar{c}_{1} \mid h\right)\right]\right) \\
& =\operatorname{sign}\left(P\left(e_{1} \mid c_{1}\right) \cdot\left[P\left(c_{1} \mid \bar{h}\right)-P\left(c_{1} \mid h\right)\right]-P\left(e_{1} \mid \bar{c}_{1}\right) \cdot\left[P\left(c_{1} \mid \bar{h}\right)-P\left(c_{1} \mid h\right)\right]\right) \\
& =\operatorname{sign}\left(\left[P\left(e_{1} \mid c_{1}\right)-P\left(e_{1} \mid \bar{c}_{1}\right)\right] \cdot\left[P\left(c_{1} \mid \bar{h}\right)-P\left(c_{1} \mid h\right)\right]\right) .
\end{aligned}
$$

By (4) we have $P\left(c_{1} \mid h\right)-P\left(c_{1} \mid \bar{h}\right)<0$ and hence the proof is completed by noting that

$$
\operatorname{sign}\left(P_{\mathcal{E}}(h)-P_{\mathcal{E}^{\prime}}(h)\right)=\operatorname{sign}\left(P\left(e_{1} \mid \bar{c}_{1}\right)-P\left(e_{1} \mid c_{1}\right)\right)
$$

Theorem 2. In case Condition B, the Ceteris Paribus Conditions and the Pairing Off Condition condition all hold, then

$$
\begin{aligned}
& \operatorname{sign}\left(P_{\mathcal{E}}(h)-P_{\mathcal{E}^{\prime}}(h)\right) \\
= & \operatorname{sign}\left(\left[P\left(e_{\left|C_{1}\right|} \mid \bar{c}_{1}\right)-P\left(e_{\left|C_{1}\right|} \mid c_{1}\right)\right] \cdot\left(\prod_{l=\left|C_{2}\right|+1}^{\left|C_{1}\right|-1} P\left(e_{l} \mid c_{1}\right)-\prod_{l=\left|C_{2}\right|+1}^{\left|C_{1}\right|-1} P\left(e_{l} \mid \bar{c}_{1}\right)\right)\right) .
\end{aligned}
$$

Proof. In a bid to further simplify notation we drop the subscript of $c$ in the following conditional probabilities: $P\left(e_{l} \mid c_{1}\right), P\left(e_{l} \mid \bar{c}_{1}\right), P\left(e_{l} \mid c_{2}\right), P\left(e_{l} \mid \bar{c}_{2}\right)$-whenever there can be no confusion. We also employ the Ceteris Paribus conditions and simply write $P(c \mid h)$ and $P(c \mid \bar{h})$; dropping the subscript of $c$.

We begin by calculating the posterior probabilities in turn

$$
\begin{aligned}
& P_{\mathcal{E}}(h)=\frac{P\left(h e_{1} \ldots e_{\left|C_{1}\right|+\left|C_{2}\right|} \vec{f}\right)}{P\left(e_{1} \ldots e_{\left|C_{1}\right|+\left|C_{2}\right|} \vec{f}\right)} \\
= & \frac{P(\vec{f} \mid h) \cdot P(h) \cdot \sum_{j, l=0}^{1} P\left(c_{1}^{j} c_{2}^{l} e_{1} \ldots e_{\left|C_{1}\right|+\left|C_{2}\right|} \mid h\right)}{\sum_{a=0}^{1} P\left(\vec{f} \mid h^{a}\right) \cdot P\left(h^{a}\right) \cdot \sum_{j, l=0}^{1} P\left(c_{1}^{j} c_{2}^{l} e_{1} \ldots e_{\left|C_{1}\right|+\left|C_{2}\right|} \mid h^{a}\right)} \\
= & \frac{P(\vec{f} \mid h) \cdot P(h) \cdot\left(\sum_{j, l=0}^{1} P\left(c^{j} \mid h\right) P\left(c^{l} \mid h\right) P\left(e_{\left|C_{1}\right|} \mid c^{j}\right) \chi_{1 j} \cdot \chi_{2 l}\right)}{\sum_{a=0}^{1} P\left(\vec{f} \mid h^{a}\right) \cdot P\left(h^{a}\right) \cdot\left(\sum_{j, l=0}^{1} P\left(c^{j} \mid h^{a}\right) P\left(c^{l} \mid h^{a}\right) P\left(e_{\left|C_{1}\right|} \mid c^{j}\right) \chi_{1 j} \cdot \chi_{2 l}\right)}
\end{aligned}
$$




$$
=\frac{1}{1+\frac{P(\vec{f} \mid \bar{h}) \cdot P(\bar{h}) \cdot\left(\sum_{j, l=0}^{1} P\left(c^{j} \mid \bar{h}\right) P\left(c^{l} \mid \bar{h}\right) P\left(e_{\left|C_{1}\right|} \mid c^{j}\right) \cdot \prod_{n=1}^{\left|C_{1}\right|-1} P\left(e_{n} \mid c^{j}\right) \cdot \prod_{g=1}^{\left|C_{2}\right|} P\left(e_{\left|C_{1}\right|+g} \mid c^{l}\right)\right)}{P(\vec{f} \mid h) \cdot P(h) \cdot\left(\sum_{j, l=0}^{1} P\left(c^{j} \mid h\right) P\left(c^{l} \mid h\right) P\left(e_{\left|C_{1}\right|} \mid c^{j}\right) \cdot \prod_{n=1}^{\left|C_{1}\right|-1} P\left(e_{n} \mid c^{j}\right) \cdot \prod_{g=1}^{\left|C_{2}\right|} P\left(e_{\left|C_{1}\right|+g} \mid c^{l}\right)\right)}} .
$$

Similarly, we find for $P_{\mathcal{E}^{\prime}}(h)$ that

$$
\begin{aligned}
& P_{\mathcal{E}^{\prime}}(h)=\frac{P^{\prime}\left(h e_{1} \ldots e_{\left|C_{1}\right|-1} e_{\left|C_{1}\right|}^{\prime} e_{\left|C_{1}\right|+1} \ldots e_{\left|C_{1}\right|+\left|C_{2}\right|} \mid \vec{f}\right)}{P\left(e_{1} \ldots e_{\left|C_{1}\right|-1} e_{\left|C_{1}\right|}^{\prime} e_{\left|C_{1}\right|+1} \ldots e_{\left|C_{1}\right|+\left|C_{2}\right|} \vec{f}\right)} \\
& =\frac{P(\vec{f} \mid h) \cdot P(h) \cdot \sum_{j, l=0}^{1} P^{\prime}\left(c_{1}^{j} c_{2}^{l} e_{1} \ldots e_{\left|C_{1}\right|-1} e_{\left|C_{1}\right|}^{\prime} e_{\left|C_{1}\right|+1} \ldots e_{\left|C_{1}\right|+\left|C_{2}\right|} \mid h\right)}{\sum_{a=0}^{1} P\left(\vec{f} \mid h^{a}\right) P\left(h^{a}\right) \sum_{j, l=0}^{1} P^{\prime}\left(c_{1}^{j} c_{2}^{l} e_{1} \ldots e_{\left|C_{1}\right|-1} e_{\left|C_{1}\right|}^{\prime} e_{\left|C_{1}\right|+1} \ldots e_{\left|C_{1}\right|+\left|C_{2}\right|} \mid h^{a}\right)} \\
& =\frac{P(\vec{f} \mid h) \cdot P(h) \cdot\left(\sum_{j, l=0}^{1} P\left(c^{j} \mid h\right) P\left(c^{l} \mid h\right) P\left(e_{\left|C_{1}\right|} \mid c^{l}\right) \cdot \chi_{1 j} \cdot \chi_{2 l}\right)}{\sum_{a=0}^{1} P\left(\vec{f} \mid h^{a}\right) \cdot P\left(h^{a}\right) \cdot\left(\sum_{j, l=0}^{1} P\left(c^{j} \mid h^{a}\right) P\left(c^{l} \mid h^{a}\right) P\left(e_{\left|C_{1}\right|} \mid c^{l}\right) \cdot \chi_{1 j} \cdot \chi_{2 l}\right)} \\
& =\frac{1}{1+\frac{P(\vec{f} \mid \bar{h}) \cdot P(\bar{h}) \cdot\left(\sum_{j, l=0}^{1} P\left(c^{j} \mid \bar{h}\right) P\left(c^{l} \mid \bar{h}\right) P\left(e_{\left|C_{1}\right|} \mid c^{l}\right) \cdot \chi_{1 j} \cdot \chi_{2 l}\right)}{P(\vec{f} \mid h) \cdot P(h) \cdot\left(\sum_{j, l=0}^{1} P\left(c^{j} \mid h\right) P\left(c^{l} \mid h\right) P\left(e_{\left|C_{1}\right|} \mid c^{l}\right) \cdot \chi_{1 j} \cdot \chi_{2 l}\right)}} .
\end{aligned}
$$

Note that the only difference between these two posteriors is that the first posterior contains the term $P\left(e_{\left|C_{1}\right|} \mid c^{j}\right)$ while the second posterior contains the term $P\left(e_{\left|C_{1}\right|} \mid c^{l}\right)$.

The leading factors play no role, the sign of $P_{\mathcal{E}}(h)-P_{\mathcal{E}^{\prime}}(h)$ is thus equal to the sign of

$$
\begin{gathered}
\frac{\sum_{j, l=0}^{1} P\left(c^{j} \mid \bar{h}\right) P\left(c^{l} \mid \bar{h}\right) P\left(e_{\left|C_{1}\right|} \mid c^{l}\right) \cdot \chi_{1 j} \cdot \chi_{2 l}}{\sum_{j, l=0}^{1} P\left(c^{j} \mid h\right) P\left(c^{l} \mid h\right) P\left(e_{\left|C_{1}\right|} \mid c^{l}\right) \cdot \chi_{1 j} \cdot \chi_{2 l}} \\
-\frac{\sum_{j, l=0}^{1} P\left(c^{j} \mid \bar{h}\right) P\left(c^{l} \mid \bar{h}\right) P\left(e_{\left|C_{1}\right|} \mid c^{j}\right) \cdot \chi_{1 j} \cdot \chi_{2 l}}{\sum_{j, l=0}^{1} P\left(c^{j} \mid h\right) P\left(c^{l} \mid h\right) P\left(e_{\left|C_{1}\right|} \mid c^{j}\right) \cdot \chi_{1 j} \cdot \chi_{2 l}} .
\end{gathered} .
$$

The sign of this expression is equal to the sign of

$$
\left(\sum_{j, l=0}^{1} P\left(c^{j} \mid \bar{h}\right) P\left(c^{l} \mid \bar{h}\right) P\left(e_{\left|C_{1}\right|} \mid c^{l}\right) \cdot \chi_{1 j} \cdot \chi_{2 l}\right)
$$




$$
\begin{gathered}
\cdot\left(\sum_{j, l=0}^{1} P\left(c^{j} \mid h\right) P\left(c^{l} \mid h\right) P\left(e_{\left|C_{1}\right|} \mid c^{j}\right) \cdot \chi_{1 j} \cdot \chi_{2 l}\right) \\
-\left(\sum_{j, l=0}^{1} P\left(c^{j} \mid \bar{h}\right) P\left(c^{l} \mid \bar{h}\right) P\left(e_{\left|C_{1}\right|} \mid c^{j}\right) \cdot \chi_{1 j} \cdot \chi_{2 l}\right) \\
\cdot\left(\sum_{j, l=0}^{1} P\left(c^{j} \mid h\right) P\left(c^{l} \mid h\right) P\left(e_{\left|C_{1}\right|} \mid c^{l}\right) \cdot \chi_{1 j} \cdot \chi_{2 l}\right) .
\end{gathered}
$$

To simplify notation we let for $j, l \in\{0,1\}$

$$
\alpha_{j l}:=\prod_{n=1}^{\left|C_{1}\right|-1} P\left(e_{n} \mid c^{j}\right) \cdot \prod_{g=1}^{\left|C_{2}\right|} P\left(e_{\left|C_{1}\right|+g} \mid c^{l}\right)=\chi_{1 j} \cdot \chi_{2 l} .
$$

We obtain the more manageable

$$
\begin{aligned}
& \left(\sum_{j, l=0}^{1} P\left(c^{j} \mid \bar{h}\right) P\left(c^{l} \mid \bar{h}\right) P\left(e_{\left|C_{1}\right|} \mid c^{l}\right) \cdot \alpha_{j l}\right) \cdot\left(\sum_{j, l=0}^{1} P\left(c^{j} \mid h\right) P\left(c^{l} \mid h\right) P\left(e_{\left|C_{1}\right|} \mid c^{j}\right) \cdot \alpha_{j l}\right) \\
- & \left(\sum_{j, l=0}^{1} P\left(c^{j} \mid \bar{h}\right) P\left(c^{l} \mid \bar{h}\right) P\left(e_{\left|C_{1}\right|} \mid c^{j}\right) \cdot \alpha_{j l}\right) \cdot\left(\sum_{j, l=0}^{1} P\left(c^{j} \mid h\right) P\left(c^{l} \mid h\right) P\left(e_{\left|C_{1}\right|} \mid c^{l}\right) \cdot \alpha_{j l}\right) .
\end{aligned}
$$

Spelling this out we obtain

$$
\begin{gathered}
\left(P(\bar{c} \mid \bar{h})^{2} P\left(e_{\left|C_{1}\right|} \mid \bar{c}\right) \alpha_{00}+P(c \mid \bar{h})^{2} P\left(e_{\left|C_{1}\right|} \mid c\right) \alpha_{11}\right. \\
\left.\quad+P(\bar{c} \mid \bar{h}) P(c \mid \bar{h})\left[P\left(e_{\left|C_{1}\right|} \mid \bar{c}\right) \alpha_{10}+P\left(e_{\left|C_{1}\right|} \mid c\right) \alpha_{01}\right]\right) \\
\cdot\left(P(\bar{c} \mid h)^{2} P\left(e_{\left|C_{1}\right|} \mid \bar{c}\right) \alpha_{00}+P(c \mid h)^{2} P\left(e_{\left|C_{1}\right|} \mid c\right) \alpha_{11}\right. \\
\left.\quad+P(\bar{c} \mid h) P(c \mid h)\left[P\left(e_{\left|C_{1}\right|} \mid c\right) \alpha_{10}+P\left(e_{\left|C_{1}\right|} \mid \bar{c}\right) \alpha_{01}\right]\right) \\
-\quad\left(P(\bar{c} \mid \bar{h})^{2} P\left(e_{\left|C_{1}\right|} \mid \bar{c}\right) \alpha_{00}+P(c \mid \bar{h})^{2} P\left(e_{\left|C_{1}\right|} \mid c\right) \alpha_{11}\right. \\
\left.\quad+P(\bar{c} \mid \bar{h}) P(c \mid \bar{h})\left[P\left(e_{\left|C_{1}\right|} \mid c\right) \alpha_{10}+P\left(e_{\left|C_{1}\right|} \mid \bar{c}\right) \alpha_{01}\right]\right) \\
\quad \cdot\left(P(\bar{c} \mid h)^{2} P\left(e_{\left|C_{1}\right|} \mid \bar{c}\right) \alpha_{00}+P(c \mid h)^{2} P\left(e_{\left|C_{1}\right|} \mid c\right) \alpha_{11}\right. \\
\left.\quad+P(\bar{c} \mid h) P(c \mid h)\left[P\left(e_{\left|C_{1}\right|} \mid \bar{c}\right) \alpha_{10}+P\left(e_{\left|C_{1}\right|} \mid c\right) \alpha_{01}\right]\right) .
\end{gathered}
$$


Fortunately, all those terms which do not contain $\alpha_{00}$ nor $\alpha_{11}$ (these are precisely those terms with $P(\bar{c} \mid \bar{h}) P(c \mid \bar{h}) P(\bar{c} \mid \bar{h}) P(c \mid \bar{h})$ ) cancel out. Furthermore, all terms which contain $\alpha_{00}^{2}$ and all terms containing $\alpha_{11}^{2}$ cancel out. Finally, all terms containing $\alpha_{00}$ and $\alpha_{11}$ also cancel out.

What remains is the much more manageable

$$
\begin{aligned}
& \left(P(\bar{c} \mid \bar{h})^{2} P\left(e_{\left|C_{1}\right|} \mid \bar{c}\right) \alpha_{00}+P(c \mid \bar{h})^{2} P\left(e_{\left|C_{1}\right|} \mid c\right) \alpha_{11}\right) \cdot \\
& \left(P(\bar{c} \mid h) P(c \mid h)\left[P\left(e_{\left|C_{1}\right|} \mid c\right) \alpha_{10}+P\left(e_{\left|C_{1}\right|} \mid \bar{c}\right) \alpha_{01}-P\left(e_{\left|C_{1}\right|} \mid \bar{c}\right) \alpha_{10}-P\left(e_{\left|C_{1}\right|} \mid c\right) \alpha_{01}\right]\right) \\
+ & \left(P(\bar{c} \mid h)^{2} P\left(e_{\left|C_{1}\right|} \mid \bar{c}\right) \alpha_{00}+P(c \mid h)^{2} P\left(e_{\left|C_{1}\right|} \mid c\right) \alpha_{11}\right) \cdot \\
& \left(P(\bar{c} \mid \bar{h}) P(c \mid \bar{h})\left[P\left(e_{\left|C_{1}\right|} \mid \bar{c}\right) \alpha_{10}+P\left(e_{\left|C_{1}\right|} \mid c\right) \alpha_{01}-P\left(e_{\left|C_{1}\right|} \mid c\right) \alpha_{10}-P\left(e_{\left|C_{1}\right|} \mid \bar{c}\right) \alpha_{01}\right]\right) \\
= & \left(P(\bar{c} \mid \bar{h})^{2} P\left(e_{\left|C_{1}\right|} \mid \bar{c}\right) \alpha_{00}+P(c \mid \bar{h})^{2} P\left(e_{\left|C_{1}\right|} \mid c\right) \alpha_{11}\right) \cdot P(\bar{c} \mid h) P(c \mid h) \\
& \cdot\left(\left[P\left(e_{\left|C_{1}\right|} \mid \bar{c}\right)-P\left(e_{\left|C_{1}\right|} \mid c\right)\right] \cdot\left[\alpha_{01}-\alpha_{10}\right]\right) \\
+ & \left(P(\bar{c} \mid h)^{2} P\left(e_{\left|C_{1}\right|} \mid \bar{c}\right) \alpha_{00}+P(c \mid h)^{2} P\left(e_{\left|C_{1}\right|} \mid c\right) \alpha_{11}\right) \cdot P(\bar{c} \mid \bar{h}) P(c \mid \bar{h}) \\
& \cdot\left(\left[P\left(e_{\left|C_{1}\right|} \mid \bar{c}\right)-P\left(e_{\left|C_{1}\right|} \mid c\right)\right] \cdot\left[\alpha_{10}-\alpha_{01}\right]\right) .
\end{aligned}
$$

We have now identified one of the factors we were looking for, $P\left(e_{\left|C_{1}\right|} \mid \bar{c}\right)-$ $P\left(e_{\left|C_{1}\right|} \mid c\right)$.

Using the Pairing Off condition we find

$$
\begin{array}{r}
\alpha_{10}-\alpha_{01}=\prod_{n=1}^{\left|C_{1}\right|-1} P\left(e_{n} \mid c\right) \cdot \prod_{g=1}^{\left|C_{2}\right|} P\left(e_{\left|C_{1}\right|+g} \mid \bar{c}\right)-\prod_{n=1}^{\left|C_{1}\right|-1} P\left(e_{n} \mid \bar{c}\right) \cdot \prod_{g=1}^{\left|C_{2}\right|} P\left(e_{\left|C_{1}\right|+g} \mid c\right) \\
=\prod_{n=1}^{\left|C_{2}\right|} P\left(e_{n} \mid c\right) \cdot \prod_{g=1}^{\left|C_{2}\right|} P\left(e_{\left|C_{1}\right|+g} \mid \bar{c}\right) \cdot\left(\prod_{l=\left|C_{2}\right|+1}^{\left|C_{1}\right|-1} P\left(e_{l} \mid c\right)-\prod_{l=\left|C_{2}\right|+1}^{\left|C_{1}\right|-1} P\left(e_{l} \mid \bar{c}\right)\right) .
\end{array}
$$

We found the second and final factor. To conclude the proof we show that

$$
\begin{aligned}
& {\left[P(\bar{c} \mid h)^{2} P\left(e_{\left|C_{1}\right|} \mid \bar{c}\right) \alpha_{00}+P(c \mid h)^{2} P\left(e_{\left|C_{1}\right|} \mid c\right) \alpha_{11}\right] \cdot P(\bar{c} \mid \bar{h}) P(c \mid \bar{h}) } \\
- & {\left[P(\bar{c} \mid \bar{h})^{2} P\left(e_{\left|C_{1}\right|} \mid \bar{c}\right) \alpha_{00}+P(c \mid \bar{h})^{2} P\left(e_{\left|C_{1}\right|} \mid c\right) \alpha_{11}\right] \cdot P(\bar{c} \mid h) P(c \mid h) } \\
> & 0 .
\end{aligned}
$$

After some algebra we find

$$
\left[P(\bar{c} \mid h)^{2} P\left(e_{\left|C_{1}\right|} \mid \bar{c}\right) \alpha_{00}+P(c \mid h)^{2} P\left(e_{\left|C_{1}\right|} \mid c\right) \alpha_{11}\right] \cdot P(\bar{c} \mid \bar{h}) P(c \mid \bar{h})
$$




$$
\begin{aligned}
& -\left[P(\bar{c} \mid \bar{h})^{2} P\left(e_{\left|C_{1}\right|} \mid \bar{c}\right) \alpha_{00}+P(c \mid \bar{h})^{2} P\left(e_{\left|C_{1}\right|} \mid c\right) \alpha_{11}\right] \cdot P(\bar{c} \mid h) P(c \mid h) \\
= & \left(P\left(e_{\left|C_{1}\right|} \mid \bar{c}\right) \alpha_{00}\right) \cdot\left[P(\bar{c} \mid h)^{2} \cdot P(\bar{c} \mid \bar{h}) P(c \mid \bar{h})-P(\bar{c} \mid \bar{h})^{2} \cdot P(\bar{c} \mid h) P(c \mid h)\right] \\
& +\left(P\left(e_{\left|C_{1}\right|} \mid c\right) \alpha_{11}\right) \cdot\left[P(c \mid h)^{2} \cdot P(\bar{c} \mid \bar{h}) P(c \mid \bar{h})-P(c \mid \bar{h})^{2} \cdot P(\bar{c} \mid h) P(c \mid h)\right] \\
= & \left(P\left(e_{\left|C_{1}\right|} \mid \bar{c}\right) \alpha_{00}\right) \cdot[P(\bar{c} \mid h) P(\bar{c} \mid \bar{h})] \cdot[P(\bar{c} \mid h) P(c \mid \bar{h})-P(\bar{c} \mid \bar{h}) P(c \mid h)] \\
& +\left(P\left(e_{\left|C_{1}\right|} \mid c\right) \alpha_{11}\right) \cdot[P(c \mid h) P(c \mid \bar{h})] \cdot[P(\bar{c} \mid \bar{h}) P(c \mid h)-P(\bar{c} \mid h) P(c \mid \bar{h})] \\
= & {[P(\bar{c} \mid \bar{h}) P(c \mid h)-P(\bar{c} \mid h) P(c \mid \bar{h})] \cdot } \\
& \left(P\left(e_{\left|C_{1}\right|} \mid c\right) \cdot \alpha_{11} \cdot P(c \mid h) \cdot P(c \mid \bar{h})-P\left(e_{\left|C_{1}\right|} \mid \bar{c}\right) \cdot \alpha_{00} \cdot P(\bar{c} \mid h) \cdot P(\bar{c} \mid \bar{h}) .\right.
\end{aligned}
$$

Since $P(c \mid h)>P(c \mid \bar{h})$ we have $P(\bar{c} \mid \bar{h})=1-P(c \mid \bar{h})>1-P(c \mid h)=P(\bar{c} \mid h)$. Thus, $P(\bar{c} \mid \bar{h}) P(c \mid h)-P(\bar{c} \mid h) P(c \mid \bar{h})>0$.

By the first Confirmatory Evidence conditions we have $P\left(e_{\left|C_{1}\right|} \mid c\right)>$ $P\left(e_{\left|C_{1}\right|} \mid \bar{c}\right)$. We also have

$$
\begin{aligned}
& \alpha_{11}-\alpha_{00}=\prod_{n=1}^{\left|C_{1}\right|-1} P\left(e_{n} \mid c_{1}\right) \prod_{g=1}^{\left|C_{2}\right|} P\left(e_{\left|C_{1}\right|+g} \mid c_{2}\right)-\prod_{n=1}^{\left|C_{1}\right|-1} P\left(e_{n} \mid \bar{c}_{1}\right) \prod_{g=1}^{\left|C_{2}\right|} P\left(e_{\left|C_{1}\right|+g} \mid \bar{c}_{2}\right) \\
& >0
\end{aligned}
$$

by the second and the third Confirmatory Evidence condition.

We thus show that

$$
\begin{aligned}
& P(c \mid h) \cdot P(c \mid \bar{h})-P(\bar{c} \mid h) \cdot P(\bar{c} \mid \bar{h}) \\
= & (1-P(\bar{c} \mid h)) \cdot(1-P(\bar{c} \mid \bar{h}))-P(\bar{c} \mid h) \cdot P(\bar{c} \mid \bar{h}) \\
= & 1-P(\bar{c} \mid h)-P(\bar{c} \mid \bar{h}) \\
> & 0 .
\end{aligned}
$$

This last inequality is (5) which we motivated above.

Corollary 2. If $E_{1}, \ldots, E_{\left|C_{1}\right|}$ are the children of $C_{1}$, then for all possible measurements $E_{1}=e_{1}, \ldots, E_{\left|C_{1}\right|}=e_{\left|C_{1}\right|}$

$$
P\left(h \mid e_{1} \ldots e_{\left|C_{1}\right|} \vec{f}\right)<P\left(h \mid c_{1} \vec{f}\right) .
$$

Proof. The proof is a relatively simple exercise in Bayesian network calculations

$$
P\left(h\left|e_{1} \ldots e_{\left|C_{1}\right|}\right| \vec{f}\right)=\frac{1}{1+\frac{P(\vec{f} \mid \bar{h}) \cdot P(\bar{h}) \cdot\left(\sum_{j=0}^{1} P\left(c^{j} \mid \bar{h}\right) \prod_{n=1}^{\left|C_{1}\right|} P\left(e_{n} \mid c^{j}\right)\right)}{P(\vec{f} \mid h) \cdot P(h) \cdot\left(\sum_{j=0}^{1} P\left(c^{j} \mid h\right) \prod_{n=1}^{\left|C_{1}\right|} P\left(e_{n} \mid c^{j}\right)\right)}} .
$$




$$
P\left(h \mid c_{1} \vec{f}\right)=\frac{1}{1+\frac{P(\vec{f} \mid \bar{h}) \cdot P(\bar{h}) \cdot(P(c \mid \bar{h}))}{P(\vec{f} \mid h) \cdot P(h) \cdot P(c \mid h)}} .
$$

Hence,

$$
\begin{aligned}
& \operatorname{sign}\left(P\left(h \mid e_{1} \ldots e_{\left|C_{1}\right|} \vec{f}\right)-P\left(h \mid c_{1} \vec{f}\right)\right) \\
= & \operatorname{sign}\left(\frac{P(c \mid \bar{h})}{P(c \mid h)}-\frac{\sum_{j=0}^{1} P\left(c^{j} \mid \bar{h}\right) \prod_{n=1}^{\left|C_{1}\right|} P\left(e_{n} \mid c^{j}\right)}{\sum_{j=0}^{1} P\left(c^{j} \mid h\right) \prod_{n=1}^{\left|C_{1}\right|} P\left(e_{n} \mid c^{j}\right)}\right) \\
= & \operatorname{sign}\left(P(c \mid \bar{h}) \sum_{j=0}^{1} P\left(c^{j} \mid h\right) \prod_{n=1}^{\left|C_{1}\right|} P\left(e_{n} \mid c^{j}\right)-P(c \mid h) \cdot \sum_{j=0}^{1} P\left(c^{j} \mid \bar{h}\right) \prod_{n=1}^{\left|C_{1}\right|} P\left(e_{n} \mid c^{j}\right)\right) \\
= & \operatorname{sign}\left(P(c \mid \bar{h})\left[P(c \mid h) \prod_{n=1}^{\left|C_{1}\right|} P\left(e_{n} \mid c\right)+P(\bar{c} \mid h) \prod_{n=1}^{\left|C_{1}\right|} P\left(e_{n} \mid \bar{c}\right)\right]\right. \\
& \left.-P(c \mid h) \cdot\left[P(c \mid \bar{h}) \prod_{n=1}^{\left|C_{1}\right|} P\left(e_{n} \mid c\right)+P(\bar{c} \mid \bar{h}) \prod_{n=1}^{\left|C_{1}\right|} P\left(e_{n} \mid \bar{c}\right)\right]\right) \\
= & \operatorname{sign}\left(P(c \mid \bar{h}) P(\bar{c} \mid h) \prod_{n=1}^{\left|C_{1}\right|} P\left(e_{n} \mid \bar{c}\right)-P(c \mid h) \cdot P(\bar{c} \mid \bar{h}) \prod_{n=1}^{\left|C_{1}\right|} P\left(e_{n} \mid \bar{c}\right)\right) \\
= & \operatorname{sign}(P(c \mid \bar{h}) P(\bar{c} \mid h)-P(c \mid h) \cdot P(\bar{c} \mid \bar{h})) \cdot
\end{aligned}
$$

By (4) we have that $P(c \mid \bar{h})<P(c \mid h)$ and that $P(\bar{c} \mid h)<P(\bar{c} \mid \bar{h})$. Hence the bracket is negative and it follows that

$$
P\left(h \mid e_{1} \ldots e_{\left|C_{1}\right|} \vec{f}\right)<P\left(h \mid c_{1} \vec{f}\right) .
$$

Proposition 2. If Condition B, the last two ceteris paribus condition and if $P\left(c_{1} \mid h\right)=1=P\left(c_{2} \mid h\right)$ hold, then

$$
\begin{aligned}
\operatorname{sign}\left(P_{\mathcal{E}}(h)-P_{\mathcal{E}^{\prime}}(h)\right)= & \operatorname{sign}\left(\frac{\sum_{j, l=0}^{1} P\left(c_{1}^{j} \mid \bar{h}\right) P\left(c_{2}^{l} \mid \bar{h}\right) P\left(e_{\left|C_{1}\right|}^{\prime} \mid c_{2}^{l}\right) \cdot \chi_{1 j} \cdot \chi_{2 l}}{P\left(e_{\left|C_{1}\right|}^{\prime} \mid c_{2}\right)}\right. \\
& \left.-\frac{\sum_{j, l=0}^{1} P\left(c_{1}^{j} \mid \bar{h}\right) P\left(c_{2}^{l} \mid \bar{h}\right) P\left(e_{\left|C_{1}\right|} \mid c_{1}^{j}\right) \cdot \chi_{1 j} \cdot \chi_{2 l}}{P\left(e_{\left|C_{1}\right|} \mid c_{1}\right)}\right) .
\end{aligned}
$$


Proof. The proof is a simple exercise in Bayesian network calculations. First, we use (11) and (12) to obtain

$$
\begin{gathered}
P_{\mathcal{E}}(h)=\frac{1}{1+\frac{P(\vec{f} \mid \bar{h}) \cdot P(\bar{h}) \cdot\left(\sum_{j, l=0}^{1} P\left(c_{1}^{j} \mid \bar{h}\right) P\left(c_{2}^{l} \mid \bar{h}\right) P\left(e_{\left|C_{1}\right|} \mid c_{1}^{j}\right) \cdot \chi_{1 j} \cdot \chi_{2 l}\right)}{P(\vec{f} \mid h) \cdot P(h) \cdot\left(\sum_{j, l=0}^{1} P\left(c_{1}^{j} \mid h\right) P\left(c_{2}^{l} \mid h\right) P\left(e_{\left|C_{1}\right|} \mid c_{1}^{j}\right) \cdot \chi_{1 j} \cdot \chi_{2 l}\right)}} . \\
P_{\mathcal{E}^{\prime}}(h)=\frac{1}{1+\frac{P(\vec{f} \mid \bar{h}) \cdot P(\bar{h}) \cdot\left(\sum_{j, l=0}^{1} P\left(c_{1}^{j} \mid \bar{h}\right) P\left(c_{2}^{l} \mid \bar{h}\right) P\left(e_{\left|C_{1}\right|}^{\prime} \mid c_{1}^{j}\right) \cdot \chi_{1 j} \cdot \chi_{2 l}\right)}{P(\vec{f} \mid h) \cdot P(h) \cdot\left(\sum_{j, l=0}^{1} P\left(c_{1}^{j} \mid h\right) P\left(c_{2}^{l} \mid h\right) P\left(e_{\left|C_{1}\right|}^{\prime} \mid c_{1}^{j}\right) \cdot \chi_{1 j} \cdot \chi_{2 l}\right)}} .
\end{gathered}
$$

Using that $P\left(c_{1} \mid h\right)=1=P\left(c_{2} \mid h\right)$ and hence $P\left(\bar{c}_{1} \mid h\right)=0=P\left(\bar{c}_{2} \mid h\right)$ the expressions simplify to

$$
P_{\mathcal{E}}(h)=\frac{1}{1+\frac{P(\vec{f} \mid \bar{h}) \cdot P(\bar{h}) \cdot\left(\sum_{j, l=0}^{1} P\left(c_{1}^{j} \mid \bar{h}\right) P\left(c_{2}^{l} \mid \bar{h}\right) P\left(e_{\left|C_{1}\right|} \mid c_{1}^{j}\right) \cdot \chi_{1 j} \cdot \chi_{2 l}\right)}{P(\vec{f} \mid h) \cdot P(h) \cdot\left(P\left(e_{\left|C_{1}\right|} \mid c_{1}\right) \cdot \chi_{1 j} \cdot \chi_{2 l}\right)}}
$$

and

$$
P_{\mathcal{E}^{\prime}}(h)=\frac{1}{1+\frac{P(\vec{f} \mid \bar{h}) \cdot P(\bar{h}) \cdot\left(\sum_{j, l=0}^{1} P\left(c_{1}^{j} \mid \bar{h}\right) P\left(c_{2}^{l} \mid \bar{h}\right) P\left(e_{\left|C_{1}\right|}^{\prime} \mid c_{1}^{j}\right) \cdot \chi_{1 j} \cdot \chi_{2 l}\right)}{P(\vec{f} \mid h) \cdot P(h) \cdot\left(P\left(e_{\left|C_{1}\right|}^{\prime} \mid c_{1}\right) \cdot \chi_{1 j} \cdot \chi_{2 l}\right)}}
$$

The claimed result follows by re-substituting the definitions of $\chi_{1 j}$ and $\chi_{2 l}$.

Proposition 3. If Condition $B$, the last two ceteris paribus condition, $P\left(c_{1} \mid h\right)=$ $1=P\left(c_{2} \mid h\right)$ and if $P\left(e_{\left|C_{1}\right|} \mid c_{1}\right)=P\left(e_{\left|C_{1}\right|}^{\prime} \mid c_{2}\right)>P\left(e_{\left|C_{1}\right|}^{\prime} \mid \bar{c}_{2}\right)$ hold, then

$$
\operatorname{sign}\left(P_{\mathcal{E}}(h)-P_{\mathcal{E}^{\prime}}(h)\right)=-\operatorname{sign}\left(\frac{\alpha_{0,0} \cdot \beta_{0,0}+\alpha_{0,1} \cdot \beta_{0,1}}{\alpha_{1,0} \cdot \beta_{1,0}}+\prod_{n=1}^{\left|C_{1}\right|-1} \frac{P\left(e_{n} \mid c_{1}\right)}{P\left(e_{n} \mid \bar{c}_{1}\right)}\right)
$$

where the $\alpha$ and $\beta$ are parameters independent of $\left|C_{1}\right|$ which are defined in (14). 
Proof. Under the assumption that $P\left(e_{\left|C_{1}\right|} \mid c_{1}\right)=P\left(e_{\left|C_{1}\right|}^{\prime} \mid c_{2}\right)$ the denominators are equal. Furthermore, the terms with $j=l=1$ cancel out. We hence find

$$
\begin{aligned}
\operatorname{sign}( & P\left(h \mid e_{1} \ldots e_{L} e_{\left|C_{1}\right|} e_{\left|C_{1}\right|+1} \ldots e_{\left|C_{1}\right|+\left|C_{2}\right|} \vec{f}\right) \\
& \left.-P\left(h \mid e_{1} \ldots e_{\left|C_{1}\right|-1} e_{\left|C_{1}\right|}^{\prime} e_{\left|C_{1}\right|+1} \ldots e_{\left|C_{1}\right|+\left|C_{2}\right|} \vec{f}\right)\right) \\
= & \operatorname{sign}\left(\sum_{\substack{j, l=0 \\
(j, l) \neq(1,1)}}^{1} P\left(c_{1}^{j} \mid \bar{h}\right) P\left(c_{2}^{l} \mid \bar{h}\right) P\left(e_{\left|C_{1}\right|}^{\prime} \mid c_{2}^{l}\right) \cdot \chi_{1 j} \cdot \chi_{2 l}\right. \\
& \left.-\sum_{\substack{j, l=0 \\
(j, l) \neq(1,1)}}^{1} P\left(c_{1}^{j} \mid \bar{h}\right) P\left(c_{2}^{l} \mid \bar{h}\right) P\left(e_{\left|C_{1}\right|} \mid c_{1}^{j}\right) \cdot \chi_{1 j} \cdot \chi_{2 l}\right) .
\end{aligned}
$$

With (subscripts represent the values for $j$ and $l$ )

$$
\begin{aligned}
& \alpha_{0,0}:=P\left(\bar{c}_{1} \mid \bar{h}\right) P\left(\bar{c}_{2} \mid \bar{h}\right) \cdot \prod_{g=1}^{\left|C_{2}\right|} P\left(e_{\left|C_{1}\right|+g} \mid \bar{c}_{2}\right) \\
& \beta_{0,0}:=P\left(e_{\left|C_{1}\right|}^{\prime} \mid \bar{c}_{2}\right)-P\left(e_{\left|C_{1}\right|} \mid \bar{c}_{1}\right) \\
& \alpha_{1,0}:=P\left(c_{1} \mid \bar{h}\right) P\left(\bar{c}_{2} \mid \bar{h}\right) \cdot \prod_{g=1}^{\left|C_{2}\right|} P\left(e_{\left|C_{1}\right|+g} \mid \bar{c}_{2}\right) \\
& \beta_{1,0}:=P\left(e_{\left|C_{1}\right|}^{\prime} \mid \bar{c}_{2}\right)-P\left(e_{\left|C_{1}\right|} \mid c_{1}\right) \\
& \alpha_{0,1}:=P\left(\bar{c}_{1} \mid \bar{h}\right) P\left(\bar{c}_{2} \mid \bar{h}\right) \cdot \prod_{g=1}^{\left|C_{2}\right|} P\left(e_{\left|C_{1}\right|+g} \mid c_{2}\right) \\
& \beta_{0,1}:=P\left(e_{\left|C_{1}\right|}^{\prime} \mid c_{2}\right)-P\left(e_{\left|C_{1}\right|} \mid \bar{c}_{1}\right)
\end{aligned}
$$

this becomes equal to

$$
\begin{aligned}
& \operatorname{sign}\left(\alpha_{0,0} \cdot \beta_{0,0} \cdot \prod_{n=1}^{\left|C_{1}\right|-1} P\left(e_{n} \mid \bar{c}_{1}\right)+\alpha_{0,1} \cdot \beta_{0,1} \cdot \prod_{n=1}^{\left|C_{1}\right|-1} P\left(e_{n} \mid \bar{c}_{1}\right)\right. \\
& \left.\quad+\alpha_{1,0} \cdot \beta_{1,0} \cdot \prod_{n=1}^{\left|C_{1}\right|-1} P\left(e_{n} \mid c_{1}\right)\right) \\
& =\operatorname{sign}\left(\left[\alpha_{0,0} \cdot \beta_{0,0}+\alpha_{0,1} \cdot \beta_{0,1}\right] \cdot \prod_{n=1}^{\left|C_{1}\right|-1} P\left(e_{n} \mid \bar{c}_{1}\right)+\alpha_{1,0} \cdot \beta_{1,0} \cdot \prod_{n=1}^{\left|C_{1}\right|-1} P\left(e_{n} \mid c_{1}\right)\right) .
\end{aligned}
$$


Note that the $\alpha$ and the $\beta$ parameters do not change value with varying $\left|C_{1}\right|$ and are hence treated as constants.

Using that $\alpha_{1,0} \cdot \beta_{1,0}<0$ and $\prod_{n=1}^{\left|C_{1}\right|-1} P\left(e_{n} \mid \bar{c}_{1}\right)>0$ we find that

$$
\begin{aligned}
\operatorname{sign}( & P\left(h \mid e_{1} \ldots e_{\left|C_{1}\right|-1} e_{\left|C_{1}\right|} e_{\left|C_{1}\right|+1} \ldots e_{\left.\left|C_{1}\right|+\left|C_{2}\right| \vec{f}\right)}\right. \\
& -P\left(h\left|e_{1} \ldots e_{\left|C_{1}\right|-1} e_{\left|C_{1}\right|}^{\prime} e_{\left|C_{1}\right|+1} \ldots e_{\left|C_{1}\right|+\left|C_{2}\right|}\right| \vec{f}\right) \\
= & -\operatorname{sign}\left(\frac{\alpha_{0,0} \cdot \beta_{0,0}+\alpha_{0,1} \cdot \beta_{0,1}}{\alpha_{1,0} \cdot \beta_{1,0}}+\frac{\prod_{n=1}^{\left|C_{1}\right|-1} P\left(e_{n} \mid c_{1}\right)}{\prod_{n=1}^{\left|C_{1}\right|-1} P\left(e_{n} \mid \bar{c}_{1}\right)}\right) .
\end{aligned}
$$

\section{B High Arity Variables}

We now address the claim that the so-far established technical results, also hold for models which employ higher arity hypothesis and/or consequence variables.

Denote by $h^{2}, h^{3}, \ldots$ the values of $H$ different from $h$ and by $c^{2}, c^{3}, \ldots$ the values of consequence variable $C$ different from $c . h^{0}$ is the (possibly infinite) disjunction of the $h^{i}$ with $i \geq 2 . c^{0}$ is the (possibly infinite) disjunction of the $c^{k}$ with $k \geq 2$. To simplify notation we let $P\left(c_{j}^{0} \mid h^{i}\right):=1-P\left(c_{j} \mid h^{i}\right)$.

The ceteris paribus conditions are that for every consequence variables $C_{j}$ and every evidence variable $E$ pertaining to the consequence variable $C_{j}$ it holds that

$$
\begin{gathered}
P\left(c_{j}^{0} \mid h^{i}\right)=P\left(c_{j}^{0} \mid h^{2}\right)=P\left(\bar{c}_{j} \mid \bar{h}\right) \\
P\left(e \mid c_{j}^{k}\right)=P\left(e \mid c_{j}^{2}\right)=P\left(e \mid \bar{c}_{j}\right) .
\end{gathered}
$$

This formalises the thought that all values of the hypothesis variable $H$ different from $h$ are equal. Furthermore, the conditional probability of the evidence does not depend on the particular value $c^{k}$.

Proposition 5. If (15) and (16) hold, then Theorem 1 and Theorem 3 hold for higher arities, too.

Proof. Using the ceteris paribus condition for the second and third equality, we find

$$
\sum_{i \geq 2} \sum_{k \geq 2} P\left(h^{i}\right) \cdot P\left(c_{j}^{k} \mid h^{i}\right) \cdot P\left(e \mid c_{j}^{k}\right)=\sum_{i \geq 2} P\left(h^{i}\right) \cdot \sum_{k \geq 2} P\left(c_{j}^{k} \mid h^{i}\right) \cdot P\left(e \mid c_{j}^{k}\right)
$$




$$
\begin{aligned}
& =P\left(e \mid c_{j}^{2}\right) \cdot \sum_{i \geq 2} P\left(h^{i}\right) \cdot \sum_{k \geq 2} P\left(c_{j}^{k} \mid h^{i}\right) \\
& =P\left(e \mid \bar{c}_{j}\right) \cdot \sum_{i \geq 2} P\left(h^{i}\right) \cdot P\left(c_{j}^{0} \mid h^{i}\right) \\
& =P\left(e \mid \bar{c}_{j}\right) \cdot P\left(\bar{c}_{j} \mid \bar{h}\right) \cdot P(\bar{h}) .
\end{aligned}
$$

To complete the proof it suffices make the following observations:

1) Whenever the term ' $P\left(e \mid \bar{c}_{j}\right) \cdot P\left(\bar{c}_{j} \mid \bar{h}\right) \cdot P(\bar{h})$ ' appears in a proof for the basic model it is replaced by the term ' $\sum_{i=2} \sum_{k=2} P\left(h^{i}\right) \cdot P\left(c_{j}^{k} \mid h^{i}\right) \cdot P\left(e \mid c_{j}^{k}\right)$ '. Since both terms are equal, no new difficulties arise.

2) The terms of the form ' $P\left(\bar{c}_{j} \mid \bar{h}\right)$ ' for $i \geq 2$ are replaced by terms of the form $\sum_{k \geq 2} P\left(c_{j}^{k} \mid h^{i}\right)^{\prime}$. The latter is equal to $P\left(c_{j}^{0} \mid h^{i}\right)$. By the first ceteris paribus assumption for greater arities, these terms are equal. 